ANDRE BROGGIN DUTRA RODRIGUES

\title{
Marcadores Prognósticos em Recém-Nascidos Portadores de Mielomeningocele
}

Dissertação apresentada à Faculdade de Medicina da Universidade de São Paulo para obtenção do título de Mestre em Ciências

Programa de Pediatria

Orientadora: Profa. Dra. Vera Lucia Jornada Krebs

São Paulo

2016 
Dados Internacionais de Catalogação na Publicação (CIP)

Preparada pela Biblioteca da

Faculdade de Medicina da Universidade de São Paulo

Creprodução autorizada pelo autor

Rodrigues, Andre Broggin Dutra

Marcadores prognósticos em recém nascidos portadores de mielomeningocele / Andre Broggin Dutra Rodrigues. -- São Paulo, 2016.

Dissertação(mestrado)--Faculdade de Medicina da Universidade de São Paulo. Programa de Pediatria.

Orientadora: Vera Lucia Jornada Krebs.

Descritores: 1.Mielomeningocele 2.Espinha bífida 3.Hidrocefalia 4.Malformação de Arnold-Chiari 5.Neurocirurgia 6.Malformações congênitas

USP/FM/DBD-012/16 


\section{DEDICATÓRIA}

À Mariana, minha esposa e fonte de inspiração;

Ao meu pai, Francisco, meu exemplo de vida e de dedicação à medicina;

À minha mãe, Maria Lúcia, retrato de ternura e compreensão, meu porto seguro;

Aos meus irmãos, Fábio e Thiago, companheiros de corpo e de alma. 


\section{AGRADECIMENTOS}

À Dra. Vera Krebs, minha orientadora, pela dedicação de tempo e sabedoria para a realização desse projeto;

Ao Dr. Hamilton Matushita, pela sua análise crítica do projeto e sugestões valiosas;

Ao Dr. Alexandre Ferraro, pelos seus ensinamentos e grande colaboração na análise estatística;

À Lucimeire Ferreira, pela pronta disponibilidade e atenção no levantamento dos prontuários do arquivo médico;

À bibliotecária Valéria Lombardi, pela revisão das referências;

Ao Josué Moreira pela edição e formatação dessa dissertação;

A todas as assistentes do Centro Neonatal do Instituto da Criança, pela minha formação acadêmica e pelo apoio pessoal. 
Essa dissertação está de acordo com as seguintes normas, em vigor no momento desta publicação:

Referências: adaptado de International Committee of Medical Journals Editors (Vancouver)

Universidade de São Paulo. Faculdade de Medicina. Serviço de Biblioteca e Documentação. Guia de apresentação de dissertações, teses e monografias. Elaborado por Anneliese Carneiro da Cunha, Maria Julia de A. L. Freddi, Maria F. Crestana, Marinalva de Souza Aragão, Suely Campos Cardoso, Valéria Vilhena. 3a ed. São Paulo: Serviço de Biblioteca e Documentação; 2011.

Abreviaturas dos títulos dos periódicos de acordo com List of Journals Indexed in Index Medicus. 


\section{SUMÁRIO}

Lista de abreviaturas

Lista de símbolos

Lista de tabelas

Resumo

Abstract

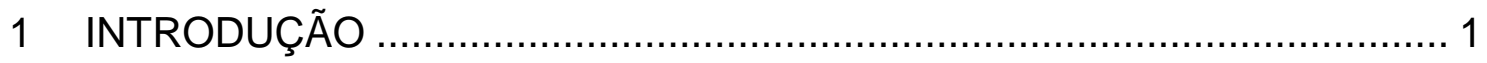

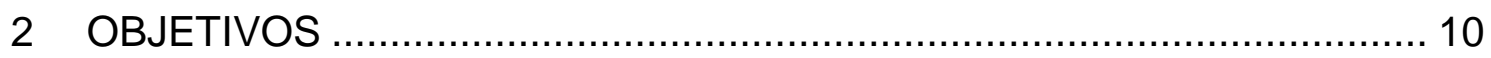

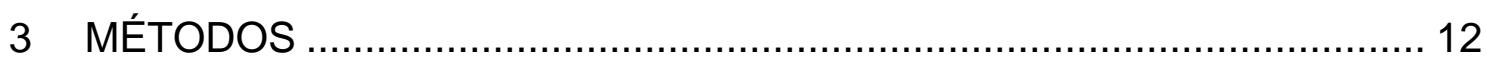

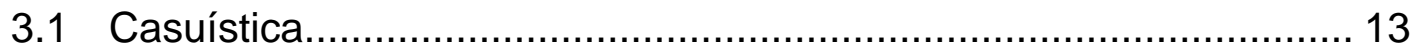

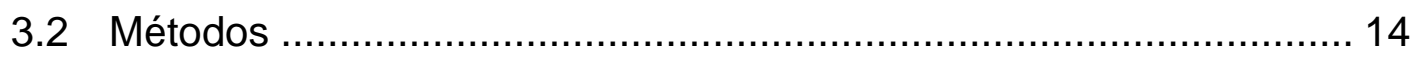

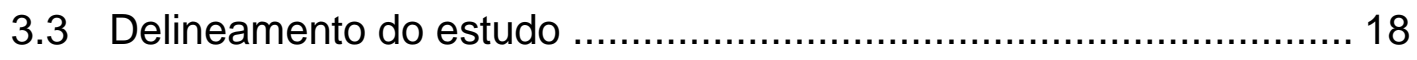

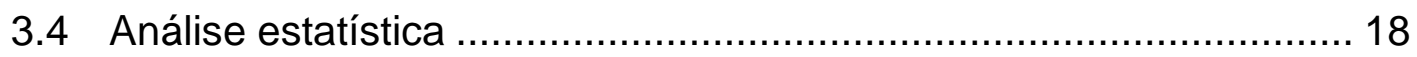

3.5 Aprovação pela Comissão de Ética............................................... 19

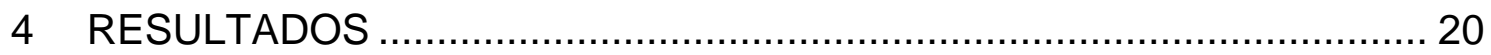

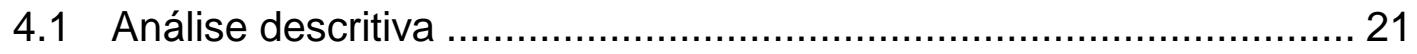

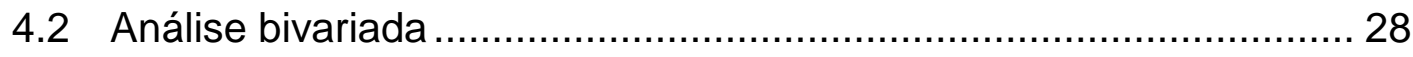

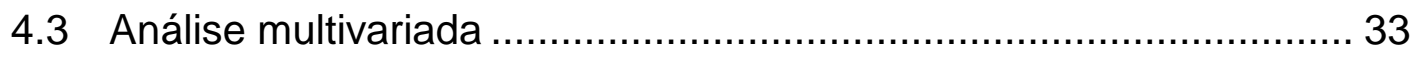

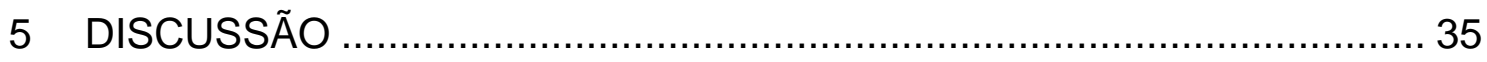

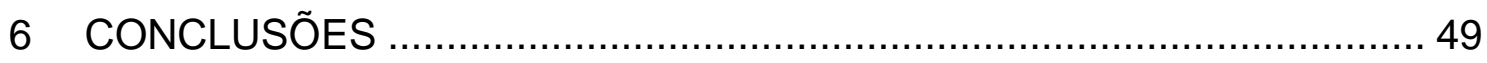

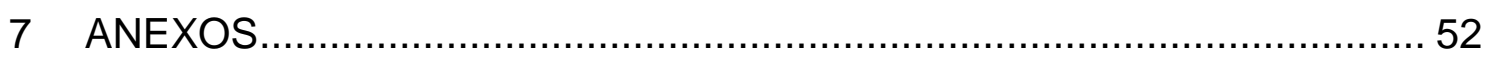

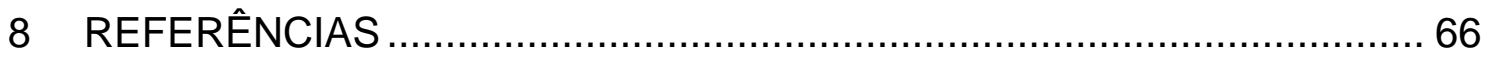




\section{LISTA DE ABREVIATURAS}

\begin{tabular}{|c|c|}
\hline MMC & - Mielomeningocele \\
\hline $\mathrm{HC}$ & - Hidrocefalia \\
\hline $\mathrm{RN}$ & - Recém-Nascidos \\
\hline $\mathrm{MCHII}$ & - Malformação Chiari do Tipo II \\
\hline LCR & - Líquido Cefalorraquidiano \\
\hline PIG & - Pequeno para Idade Gestacional \\
\hline AIG & - Adequado para Idade Gestacional \\
\hline GIG & - Grande para Idade Gestacional \\
\hline PC & - Perímetro Cefálico \\
\hline PN & - Peso de Nascimento \\
\hline DM & - Diabetes Melitus \\
\hline RPMO & - Rotura Prematura de Membranas Ovulares \\
\hline TPP & - Trabalho de Parto Prematuro \\
\hline SFA & - Sofrimento Fetal Agudo \\
\hline DHEG & - Doença Hipertensiva Específica da Gestação \\
\hline IG & - Idade Gestacional \\
\hline VPP & - Ventilação Com Pressão Positiva \\
\hline DVP & - Derivação Ventrículo Peritoneal \\
\hline DVE & - Derivação Ventricular Externa \\
\hline FO & - Ferida Operatória \\
\hline \multirow[t]{2}{*}{ ICrHCFMUSP } & - Instituto da Criança do Hospital das Clínicas da Faculdade de \\
\hline & Medicina da Universidade de São Paulo \\
\hline FDA & - "Food and Drug Administration" \\
\hline VS. & - Versus \\
\hline IC & - Intervalo de Confiança \\
\hline $\mathrm{RR}$ & - Risco Relativo \\
\hline IRR & - "Incidence Rate Ratio" \\
\hline $\mathrm{RH}$ & - Registro Hospitalar \\
\hline No & - Número \\
\hline
\end{tabular}




\section{LISTA DE SÍMBOLOS}

$$
\begin{array}{ll}
< & \text { - Menor que } \\
> & \text { - Maior que } \\
\leq & \text { - Menor ou igual } \\
\geq & \text { - Maior ou igual } \\
\% & \text { - Porcentagem } \\
+ & \text { - Adição } \\
- & \text { - Subtração } \\
= & \text { - Igual a } \\
\text { / } & \text { - Divisão } \\
\mathrm{g} & \text { - Gramas } \\
\mathrm{Kg} & \text { - Kilogramas } \\
\mathrm{p} & \text { - Índice de probabilidade } \\
\mathrm{N} & \text { - Tamanho da amostra } \\
\mathrm{B} & \text { - Coeficiente de regressão } \\
\mathrm{Cm} & \text { - Centímetros } \\
\mathrm{M} & \text { - Masculino } \\
\mathrm{F} & \text { - Feminino } \\
\mathrm{S} & \text { - Sim } \\
\mathrm{N} & \text { - Não } \\
\hline
\end{array}
$$




\section{LISTA DE TABELAS}

Tabela 1 - Incidência de MMC entre os nascidos vivos no Centro Neonatal do ICrHCFMUSP por ano de estudo

Tabela 2 - Incidência de malformações e síndromes genéticas associadas à $\mathrm{MMC}$ em 9 neonatos excluídos do estudo

Tabela 3 - Estatística descritiva das características maternas......................23

Tabela 4 - Estatística descritiva das características dos RN.........................24

Tabela 5 - Estatística descritiva das características anatômicas da MMC....25

Tabela 6 - Estatística descritiva das características da correção cirúrgica

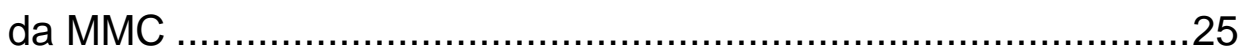

Tabela 7 - Estatística descritiva do atendimento em sala de parto ...............26

Tabela 8 - Estatística descritiva dos desfechos de curto prazo analisados durante internação hospitalar..................................................27

Tabela 9 - Análise bivariada dos marcadores prognósticos vs. VPP e

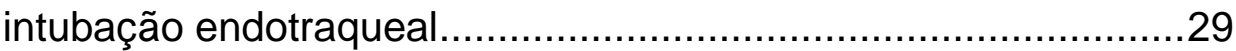

Tabela 10 - Análise bivariada dos marcadores prognósticos vs. tempo de

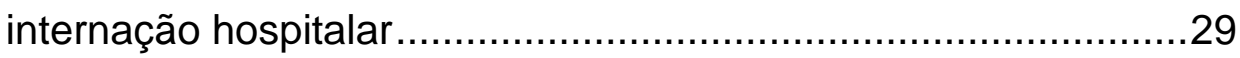

Tabela 11 - Análise bivariada dos marcadores prognósticos vs. necessidade de derivação ventricular

Tabela 12 - Análise bivariada dos marcadores prognósticos vs. infecção e deiscência de FO

Tabela 13 - Análise bivariada dos marcadores prognósticos vs. infecção de SNC e sepse

Tabela 14 - Análise bivariada dos marcadores prognósticos vs. desfecho combinado de evolução favorável.

Tabela 15 - Análise multivariada dos marcadores prognósticos vs. desfechos de curto prazo nos pacientes com MMC 


\section{RESUMO}

Rodrigues ABD. Marcadores Prognósticos em Recém-Nascidos Portadores de Mielomeningocele [dissertação]. São Paulo: Faculdade de Medicina, Universidade de São Paulo; 2016. 75p.

Introdução: Pacientes com mielomeningocele apresentam elevada mortalidade e desenvolvem déficits neurológicos que ocorrem, primariamente, pelo desenvolvimento anormal da medula e de raízes nervosas e, secundariamente, por complicações adquiridas no período pós-natal. O desafio no cuidado desses pacientes é o reconhecimento precoce dos recém-nascidos de risco para evolução desfavorável a fim de estabelecer estratégias terapêuticas individualizadas. Objetivo: Este estudo tem como objetivo identificar marcadores prognósticos de curto prazo para recém-nascidos com mielomeningocele. As características anatômicas do defeito medular e da sua correção neurocirúrgica foram analisadas para esta finalidade. Métodos: Foi realizado um estudo de coorte retrospectiva com 70 pacientes com mielomeningocele em topografia torácica, lombar ou sacral nascidos entre janeiro de 2007 a dezembro de 2013 no Centro Neonatal do Instituto da Criança do Hospital das Clínicas da Faculdade de Medicina da Universidade de São Paulo. Pacientes com infecção congênita, anomalias cromossômicas e outras malformações maiores não relacionadas à mielomeningocele foram excluídos da análise. As características anatômicas da mielomeningocele e a sua correção neurocirúrgica foram analisadas quanto aos seguintes desfechos: reanimação neonatal, tempo de internação, necessidade de derivação ventricular, deiscência da ferida operatória, infecção da ferida operatória, infecção do sistema nervoso central e sepse. Para a análise bivariada dos desfechos qualitativos com os fatores de interesse foram empregados testes do qui-quadrado e exato de Fisher. Para a análise do desfecho quantitativo, tempo de internação hospitalar, foram empregados testes de Mann-Whitney. Foram estimados os riscos relativos e os respectivos intervalos com $95 \%$ de confiança. Foram desenvolvidos modelos de regressão linear múltipla para os desfechos quantitativos e regressão de Poisson para os desfechos qualitativos. Resultados: Durante o período do estudo 12.559 recém-nascidos foram admitidos no Centro Neonatal do Instituto da Criança do Hospital das Clínicas da Faculdade de Medicina da Universidade de São Paulo. Oitenta pacientes foram diagnosticados com mielomeningocele, com incidência de 6,4 casos para cada 1.000 nascidos vivos. Dez pacientes foram excluídos da análise devido à mielomeningocele em topografia cervical $(n=1)$, à cardiopatia congênita $(n=4)$, à trissomia do cromossomo $13(n=1)$, à onfalocele $(n=3)$ e à encefalocele $(n=1)$. Ocorreram três óbitos $(4,28 \%)$. Mielomeningocele extensa foi associada a infecção do sistema nervoso central, a complicação de 
ferida operatória e a maior tempo de internação hospitalar. Os pacientes com mielomeningocele em topografia torácica apresentaram tempo de internação, em média, 39 dias maior que aqueles com defeito em topografia lombar ou sacral. Houve maior necessidade de reanimação em sala de parto entre os pacientes com macrocrania ao nascer. A correção cirúrgica realizada após 48 horas de vida aumentou em 5,7 vezes o risco de infecção do sistema nervoso central. Entre os pacientes operados nas primeiras 48 horas de vida não foi observado benefício adicional na correção cirúrgica realizada em "tempo zero". A ausência de hidrocefalia antenatal foi um marcador de bom prognóstico. Nestes pacientes, a combinação dos desfechos necessidade de derivação ventricular, complicações infecciosas, complicações de ferida operatória e reanimação em sala de parto foi $70 \%$ menos frequente. Conclusão: Este estudo permitiu identificar marcadores prognósticos de curto prazo em recémnascidos com mielomeningocele. Os defeitos medulares extensos e a correção cirúrgica após 48 horas de vida influenciaram negativamente na evolução de curto prazo. As lesões extensas foram associadas a maiores taxas de infecção do sistema nervoso central, a complicações de ferida operatória e a internação hospitalar prolongada. A correção cirúrgica realizada após 48 horas de vida aumentou significativamente a ocorrência de infecção do sistema nervoso central. Ausência de hidrocefalia antenatal foi associada a menor número de complicações nos primeiros dias de vida.

Descritores: 1.Mielomeningocele, 2.Espinha bífida, 3.Hidrocefalia, 4.Malformação de Arnold-chiari, 5.Neurocirurgia, 6.Malformações congênitas 


\begin{abstract}
Rodrigues ABD. Short-term prognostic factors in myelomeningocele patients [dissertation]. São Paulo: "Faculdade de Medicina, Universidade de São Paulo"; 2016. 75p.
\end{abstract}

Introduction: Patients with myelomeningocele have a high mortality and develop neurological disabilities that occur primarily by a defective spinal cord and nerve root development and, secondarily, by acquired post-natal complications. The challenge in the post-natal management of myelomeningocele is the early recognition of cases at risk for complications in order to establish individualized treatment strategies. Objective: This study aims to identify short-term prognostic markers for newborns with myelomeningocele. Anatomical characteristics of the spinal defect and technical aspects of the neurosurgical repair were analyzed for this purpose. Methods: A retrospective cohort study was conducted in 70 patients with thoracic, lumbar or sacral myelomeningocele born between January 2007 and December 2013 in the Centro Neonatal do Instituto da Criança do Hospital das Clínicas da Faculdade de Medicina da Universidade de São Paulo. Patients with congenital infection, chromosomal abnormalities and other major malformations unrelated to myelomeningocele were excluded from our analysis. Features of myelomeningocele anatomy and neurosurgical treatment were analyzed for the following outcomes: neonatal resuscitation, length of hospital stay, need for ventricular shunt, wound dehiscence, wound infection, central nervous system infection and sepsis. Relationships between qualitative outcomes and factors of interest were examined using chi-square or Fisher's exact tests. The relationships with the quantitative outcome duration of hospital stay were evaluated using the MannWhitney tests. Relative risks were estimated with $95 \%$ confidence intervals. Multivariate linear regression was used to evaluate the quantitative outcomes and a Poisson regression model was used for the qualitative outcomes. Results: During the study period a total of 12,559 neonates were born in Centro Neonatal do Instituto da Criança do Hospital das Clínicas da Faculdade de Medicina da Universidade de São Paulo. Eighty patients were diagnosed with myelomeningocele resulting in an incidence of 6.4 cases per 1000 live births. Ten patients were excluded from our analysis due to cervical myelomeningocele $(n=1)$, congenital heart disease $(n=4)$, trisomy $13(n=1)$, omphalocele $(n=3)$ and encephalocele $(n=1)$. Three deaths were observed in the study period $(4,28 \%)$. Large myelomeningocele was associated with central nervous system infection, wound complications and longer hospital stay. Patients with thoracic myelomeningocele required longer hospital stay, on average 39 days longer when compared to patients with lumbar or sacral defects. There was a positive correlation between the need for resuscitation at 
the delivery room and the presence of macrocrania at birth. Late surgical repair performed after 48 hours of life increased in 5.7 times the risk of central nervous system infection. Among patients operated within the first 48 hours, no additional benefit in interventions held in "time zero" was observed. Absence of antenatal hydrocephalus was a favorable prognostic marker. In these cases, the combination of need for ventricular drainage, sepsis, central nervous system infection, complications of surgical site and intervention in the delivery room were $70 \%$ lower. Conclusion: This study allowed us to identify short-term prognostic markers for newborns with myelomeningocele. Extensive spinal cord defect and surgical repair after 48 hours of life negatively influenced short-term outcomes. Extensive lesions were associated with higher rates of central nervous system infections, surgical wound complications and prolonged hospital stay. Interventions performed 48 hours after birth significantly increased occurrence of central nervous system infections. Absence of antenatal hydrocephalus was associated with fewer complications in the first days of life.

Descriptors: 1.Myelomeningocele, 2.Spina bifida, 3 Hydrocephalus, 4.Arnold-chiari malformation, 5.Neurosurgery, 6.Congenital malformations 
As malformações congênitas são anomalias morfológicas, funcionais ou metabólicas presentes ao nascimento, secundárias a causas genéticas, ambientais ou mistas ${ }^{(1)}$. Aproximadamente $3 \%$ dos recém-nascidos (RN) são portadores de uma ou mais malformações e $7 \%$ das mortes neonatais estão relacionadas a essas anomalias ${ }^{(2)}$.

Os defeitos de fechamento do tubo neural são malformações congênitas do sistema nervoso central (SNC) com alta prevalência populacional. É a segunda malformação mais frequente com prevalência de 0,5 a 8 casos para cada 1.000 nascidos vivos ${ }^{(3)}$.

A neurulação é o processo embriônico de formação do tubo neural a partir do tecido neuroectodérmico que ocorre entre a terceira e a sexta semana de gestação. Nele, eventos sequenciais de migração e diferenciação celular se sucedem sob a ação sincronizada de genes reguladores e intensa atividade metabólica. A interferência nesse processo ocasiona uma falha de fechamento do tubo neural com alterações do SNC e estruturas adjacentes, como meninges, coluna vertebral e tecido cutâneo ${ }^{(4)}$.

O defeito de fechamento do tubo neural ocorre como parte de síndromes genéticas, em associação com alterações cromossômicas e relacionado a exposições ambientais ${ }^{(5)}$. Mas, na maioria dos casos, apresenta-se como malformação isolada de origem multifatorial ${ }^{(6)}$. 
Os fatores ambientais são responsáveis por aproximadamente 0,12 casos de malformação congênita para cada 1.000 nascimentos $^{(7)}$. As principais exposições ambientais implicadas no desenvolvimento anormal do tubo neural são: exposição materna ao ácido valpróico ${ }^{(8)}$, diabetes materno ${ }^{(9)}$, obesidade materna $^{(10)}$ e exposição materna a altas temperaturas no início da gestação ${ }^{(5)}$.

Quanto às evidências do envolvimento de fatores genéticos na etiologia dessas malformações podemos citar: o risco aumentado de recorrência entre familiares, o risco de recorrência em gestações de mulheres com fetos acometidos e a associação com síndromes conhecidas e outras malformações, como síndrome de meckel, estenose anal e trissomias dos cromossomos 13 e $18^{(11)}$.

A deficiência de folato parece desempenhar um papel fundamental na etiologia de várias malformações congênitas. O folato atua em processos bioquímicos e reações metabólicas essenciais ao crescimento e diferenciação dos tecidos. Participa da proliferação celular com envolvimento na síntese de pirimidinas e purinas e fornece grupos metil para macromoléculas como DNA, proteínas e lipídios ${ }^{(12)}$. A regulação da expressão gênica, comandada pela metilação do DNA, é responsável por eventos celulares como proliferação, diferenciação, migração e morte celular. Também controla a síntese de proteínas e é essencial para a produção de membranas plasmáticas.

A deficiência de folato é particularmente importante durante a embriogênese, pois interferências ambientais e alterações genéticas implicadas no metabolismo do folato aumentam o risco de malformações ${ }^{(13)}$. Na deficiência de folato a replicação celular é afetada, ocorre hipometilação do DNA e muitos 
processos bioquímicos são interrompidos. A perda da regulação epigenética durante o processo de neurulação interfere com o crescimento normal dos tecidos $^{(14)}$. Cerca de $70 \%$ dos casos de mielomeningocele (MMC) são potencialmente preveníveis com a suplementação periconcepcional de ácido fólico ${ }^{(15)}$.

A MMC é o subtipo de defeito de fechamento do tubo neural mais frequente, com alteração significativa do desenvolvimento neurológico no âmbito anatômico e funcional. A lesão pode ser plana e displásica ou manter parcialmente o aspecto tubular da medula com maior acometimento da sua porção dorsal. Na maioria dos casos, através da falha de fechamento da coluna vertebral posterior, há uma herniação de conteúdo cístico composto por tecido neural associado ao aumento do espaço subaracnóideo ventral ao componente medular ${ }^{(16)}$.

A variação temporal e geográfica na prevalência da $M M C$ é bem documentada na literatura ${ }^{(17)}$. Está relacionada a aspectos étnicos, nutricionais e à possibilidade de interrupção eletiva da gestação em alguns países ${ }^{(18)}$. Nos Estados Unidos, a prevalência de MMC ficou estável em 0,34 casos para cada 1.000 nascidos vivos após política de fortificação de alimentos com ácido fólico ${ }^{(19)}$. No Brasil, séries de casos em regiões específicas apresentam incidências de 0,6 a 1,13 casos para cada 1.000 nascidos vivos ${ }^{(20,21)}$. No Centro Neonatal do Instituto da Criança do Hospital das Clínicas da Faculdade de Medicina da Universidade de São Paulo (ICrHCFMUSP), no período de janeiro de 2006 a junho de 2007, a incidência de malformações do SNC foi de 11,4 para cada 1.000 nascidos vivos. MMC foi a principal malformação descrita com incidência de 3,6 casos para 1.000 nascidos vivos ${ }^{(22)}$. 
Nos pacientes com MMC a evolução clínica de curto e longo prazo é o resultado de diversos fatores que determinam o dano neuronal e interferem no desenvolvimento do SNC. A gravidade depende da localização, do conteúdo e da extensão do defeito medular ${ }^{(23)}$. Alterações e danos estruturais ao SNC ocorrem no momento da embriogênese, no período intrauterino e continuam na vida pós-natal.

De acordo com a teoria da dupla lesão, a disfunção neurológica ocorre inicialmente pela falha de fechamento do tubo neural, a não neurulação, seguida de um processo neurodegenerativo medular, denominada segunda lesão ${ }^{(24)}$. A formação anatômica incompleta das estruturas que protegem a medula espinhal expõe os tecidos a um ambiente hostil com predisposição a danos $^{(25)}$. A lesão secundária ocorre quando a medula e raízes nervosas sofrem deterioração pela exposição ao líquido amniótico, ao trauma direto e aos eventos infecciosos.

O acometimento do SNC não está restrito ao componente medular. Outras anomalias se relacionam ao defeito primário com graus variáveis de acometimento encefálico, como a hidrocefalia $(\mathrm{HC})$ e a malformação de Chiari do tipo II (MChII) ${ }^{(26)}$.

A HC ocorre quando há um acúmulo excessivo de LCR nos ventrículos ou no espaço subaracnóideo, decorrente de uma alteração na sua formação, circulação ou reabsorção ${ }^{(27)}$. Acomete 65 a 93\% dos pacientes com MMC e é secundária à obstrução ao fluxo liquórico na topografia da fossa posterior com subsequente dilatação dos ventrículos cerebrais ${ }^{(28)}$. 
A apresentação clínica da HC é variável, sendo os sinais e sintomas de hipertensão intracraniana os mais frequentes ${ }^{(29)}$. $O$ efeito compressivo do sistema ventricular em expansão sobre as estruturas adjacentes implica em alterações motoras, alterações de pares cranianos e déficits cognitivos no longo prazo ${ }^{(30)}$.

Nesses pacientes é frequente a necessidade de derivação ventricular para proteção cerebral contra os efeitos deletérios da HC progressiva. A necessidade deste procedimento está relacionada à topografia da lesão medular, sendo as lesões pequenas e de topografia sacral as de menor probabilidade para evoluir com HC progressiva ${ }^{(31)}$. O desvio do fluxo liquórico através da inserção de derivações ventriculares reduz a dilatação dos ventrículos, melhora a função neurológica e o prognóstico intelectual ${ }^{(32)}$. Há redução do edema de substância branca com ganho correspondente de espessura cortical. A melhora clínica é resultado da melhora funcional dos neurônios remanescentes, sendo possível a recuperação apenas até determinado estágio de agressão(33).

A detecção precoce da $\mathrm{HC}$ e a rápida instalação de derivação ventricular são de grande importância para obter um melhor resultado neurológico. Contudo, como o procedimento pode apresentar complicações, há controvérsias quanto ao melhor momento para a sua realização e se este deve ou não ser feito no mesmo tempo cirúrgico da correção do defeito medular ${ }^{(28)}$.

A MChll é uma alteração congênita complexa com extenso acometimento das estruturas da fossa posterior. Envolve o deslocamento caudal da medula, quarto ventrículo e cerebelo através do forame occipital ${ }^{(34)}$. 
É responsável pela maioria dos óbitos nos pacientes com $\mathrm{MMC}^{(35,36)}$. Ocorre em até $90 \%$ dos pacientes, porém se apresenta de forma sintomática em apenas 15 a $35 \%$ dos casos $^{(37)}$. Entre os RN, manifesta-se habitualmente com episódios de apneia, estridor e dificuldade de deglutição. Outros sintomas incluem dificuldade de sucção, cianose e refluxo gastroesofágico ${ }^{(38,39)}$. Nas crianças maiores o efeito compressivo medular tem como apresentação mais comum a fraqueza e as alterações sensoriais de extremidades ${ }^{(40)}$.

A apresentação clínica está relacionada ao comprometimento do tronco cerebral e dos pares cranianos. A inserção de derivação ventricular e a descompressão da fossa posterior resultam em melhora evolutiva nos casos em que o comprometimento neurológico é secundário ao efeito compressivo da HC e à tração de raízes nervosas ${ }^{(41)}$. Contudo, a resposta ao tratamento cirúrgico é precária quando o dano neurológico já está estabelecido, quando a isquemia associada à compressão cervicomedular é irreversível e quando há disgenesia do tronco cerebral ${ }^{(42)}$.

As principais causas de morbimortalidade entre os pacientes com MMC são disfunção de tronco cerebral, infecção de SNC e complicações relacionadas à intervenção neurocirúrgica. A otimização dos cuidados pós natais destes pacientes possibilitou melhora na sobrevida com aumento significativo de crianças que atingem a idade $\operatorname{adulta}^{(18)}$.

A redução da morbidade é secundária aos avanços no tratamento neurocirúrgico da MMC e das malformações do SNC associadas, como a intervenção cirúrgica precoce ${ }^{(43)}$ e a implantação de derivação ventricular para tratamento da $\mathrm{HC}^{(44)}$. $\mathrm{O}$ momento da correção cirúrgica parece alterar os 
desfechos de curto e longo prazo, sendo que a intervenção imediata após o nascimento demonstrou superioridade quanto à deiscência de ferida operatória (FO) e neurodesenvolvimento com um ano de vida ${ }^{(45)}$.

A correção cirúrgica intrauterina tem sido praticada em alguns centros nos últimos 15 anos $^{(46)}$. Oferece a possibilidade de reduzir a incidência de complicações, com menor lesão medular secundária, redução na incidência de MChll e redução da necessidade de derivação ventricular para tratamento da $\mathrm{HC}^{(47)}$. No entanto, o custo elevado do procedimento antenatal, os riscos para a gestante e os eventos complicadores para a atual e futuras gestações impedem a ampla utilização desse procedimento.

O Colégio Americano de Obstetrícia e Ginecologia recomenda que a cirurgia fetal seja apenas oferecida em centros com experiência nesse tipo de procedimento, com envolvimento de equipe multidisciplinar treinada e com instalações capazes de oferecer o tratamento intensivo necessário ${ }^{(48)}$.

\section{Justificativa}

A relevância da $M M C$ no contexto de saúde pública pode ser mensurada pelo seu impacto social em termos de mortalidade, morbidade e custo de tratamento. Para o indivíduo, o impacto da doença reflete-se em incapacidade funcional, dor, anos potenciais de vida perdidos e custo familiar. O desafio no cuidado destes pacientes está em oferecer soluções assistenciais ao indivíduo e simultaneamente contemplar as demandas sociais de uma doença complexa. 
Em nosso meio há poucos estudos com objetivo de avaliar a evolução inicial da MMC, do nascimento até o momento da alta hospitalar. Consideramos importante identificar as complicações apresentadas por estes $\mathrm{RN}$ e as intercorrências cirúrgicas determinantes de desfechos desfavoráveis durante a internação. Este conhecimento poderá contribuir para a predição da evolução clínica e permitirá otimizar os cuidados pós-natais com melhora na sobrevida e redução na morbidade desses neonatos.

\section{Hipótese}

A hipótese do estudo é que a análise das características anatômicas do defeito medular e da sua correção neurocirúrgica permitirá identificar marcadores prognósticos em RN com MMC. 
2 OBJETIVOS 
O presente estudo tem como objetivos:

1. Verificar a incidência de $M M C$ entre os pacientes admitidos no Centro Neonatal do ICrHCFMUSP no período de sete anos.

2. Identificar e analisar marcadores de risco para desfechos desfavoráveis de curto prazo em RN com MMC. 
3 MÉTODOS 


\subsection{Casuística}

\section{Modelo do Estudo}

O modelo do estudo foi observacional através da análise de uma coorte retrospectiva de neonatos com MMC. Foram avaliados todos os pacientes com diagnóstico de MMC nascidos no período de janeiro de 2007 a dezembro de 2013, no Centro Neonatal do ICrHCFMUSP, centro de referência nacional de gestação de alto risco.

\section{Critérios de inclusão}

Foram incluídos no estudo todos os RN diagnosticados com MMC em topografia torácica, lombar ou sacral admitidos no Centro Neonatal do ICrHCFMUSP no período do estudo.

\section{Critérios de exclusão}

Foram excluídos os neonatos que apresentarem as seguintes condições:

- MMC em topografia cervical

- Infecções congênitas

- Anormalidades cromossômicas

- Outras malformações maiores não relacionadas à MMC

- Nascidos de gestação complicada com corioamnionite. 


\subsection{Métodos}

Foi realizada a descrição de características anatômicas do defeito medular e do tratamento neurocirúrgico como marcadores prognósticos evolutivos de RN com MMC.

As características anatômicas da MMC consideradas como marcadores de prognóstico de curto prazo foram:

- Topografia do defeito medular

- Extensão da lesão

- Presença de HC antenatal

- Macrocrania ao nascimento

- Perda da integridade das membranas que recobrem o defeito medular.

As características do tratamento neurocirúrgico de correção da MMC consideradas como marcadores de prognóstico de curto prazo foram:

- Momento da correção cirúrgica

- Correção cirúrgica imediata ao nascimento.

Os marcadores de prognóstico foram analisados quanto a:

- Necessidade de reanimação neonatal

- Tempo de internação hospitalar

- Necessidade de derivação ventricular

- Deiscência de FO 
- Infecção de FO

- Infecção de SNC

- Sepse

Para a criação do banco de dados foi feita a revisão de prontuários e preenchido um formulário eletrônico com dados antenatais, dados do parto, dados neonatais e tratamento clínico e cirúrgico instituído em cada recém-nascido.

O diagnóstico de MMC foi determinado pelo neonatologista no nascimento, considerando-se o acometimento medular e de tecidos adjacentes através de um defeito de fechamento da coluna vertebral posterior. As características anatômicas do defeito medular foram interpretadas através da análise descritiva da lesão realizada pelo neonatologista em sala de parto e pelo neurocirurgião no momento da correção cirúrgica.

Os defeitos medulares foram classificados em:

- Não extensos, quando foi possível realizar o fechamento primário da FO.

- Extensos, quando foi necessário utilizar técnicas de retalho cirúrgico para o fechamento livre de tensão da FO.

Macrocrania foi definida quando o perímetro cefálico (PC) aferido foi superior ao percentil 97 para a idade gestacional de nascimento (IG) segundo curvas de crescimento de Fenton ${ }^{(49)}$. A aferição do PC foi realizada em sala de parto, circundando-se o crânio no plano horizontal com fita métrica e utilizando como pontos de referência a glabela anteriormente e a protuberância occipital posteriormente. 
A descrição de drenagem espontânea de LCR determinou a perda da integridade das membranas. A derivação ventricular foi indicada:

- Na presença de drenagem persistente de LCR pela FO

- Na presença de fontanela abaulada e tensa

- $\mathrm{Na}$ presença de dilatação progressiva do sistema ventricular em exames ultrassonográficos seriados

A correção da MMC no "tempo zero" foi definida quanto o procedimento cirúrgico foi realizado imediatamente após o nascimento, antes da admissão em unidade de terapia intensiva. Todos os pacientes receberam profilaxia com antibiótico iniciada precocemente.

Foi considerada intervenção em sala de parto quando houve suporte ventilatório ao RN com, ao menos, ventilação com pressão positiva (VPP).

O diagnóstico de ventriculite foi determinado ${ }^{(50)}$ :

- Quando houve isolamento de microrganismo na cultura ou no exame bacterioscópico do LCR

- Quando o paciente apresentou sintomas de infecção e alterações no exame do LCR (aumento de leucócitos, aumento da concentração de proteínas, diminuição da concentração de glicose).

Sepse neonatal foi considerada se o paciente apresentou síndrome da resposta inflamatória sistêmica associada à infecção presumida ou na presença de sintomas com hemocultura positiva ${ }^{(51)}$.

O diagnóstico de infecção de FO foi determinado ${ }^{(50)}$ : 
- Pela presença de drenagem purulenta da incisão cirúrgica

- Pela necessidade de reabordagem cirúrgica no caso de febre e sinais inflamatórios locais

- Pelo achado de abscesso no intra-operatório ou no exame histopatológico

- Na suspeita de infecção pelo neurocirurgião.

Foram considerados de evolução favorável aqueles pacientes que no período de internação não necessitaram de suporte em sala de parto, não foram submetidos à derivação ventricular e não evoluíram com complicações infecciosas ou complicações relacionadas à FO. 


\subsection{Delineamento do estudo}

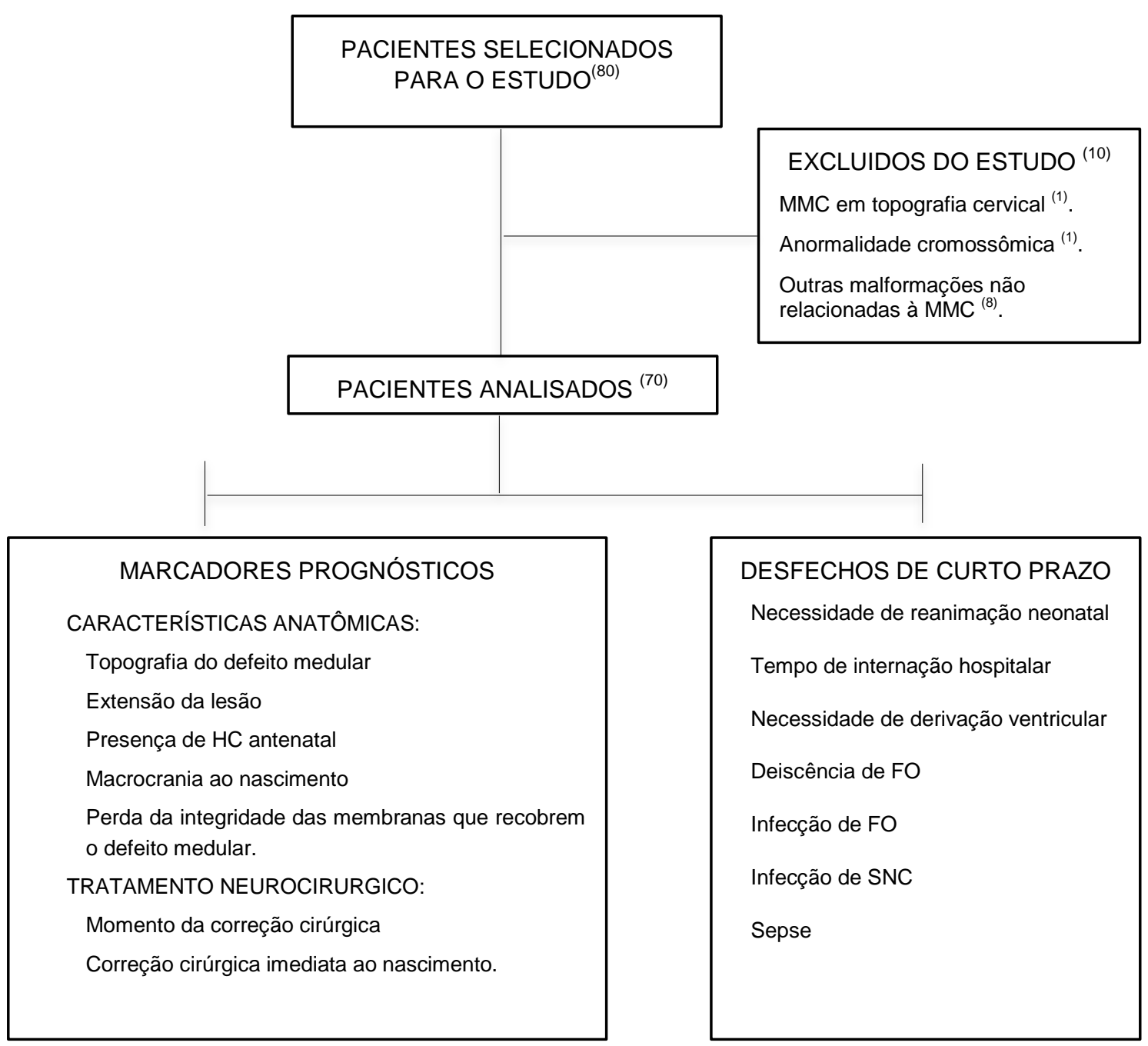

\subsection{Análise estatística}

As características quantitativas pessoais e clínicas da gestante e do RN foram descritas com uso de medidas resumo (média, desvio padrão, mediana, mínimo e máximo) e as características qualitativas foram descritas com uso de frequências absolutas e relativas. 
Cada desfecho qualitativo avaliado foi relacionado aos fatores de interesse através de testes de associação, teste do qui-quadrado ou teste exato de Fisher ${ }^{(52)}$. O desfecho quantitativo tempo de internação foi comparado entre as categorias dos marcadores de prognóstico com testes MannWhitney ${ }^{(52)}$. Foram estimados os riscos relativos e os respectivos intervalos com $95 \%$ de confiança de cada marcador de prognóstico com os desfechos ${ }^{(53)}$.

Para a análise multivariada foram desenvolvidos modelos de regressão de Poisson para os desfechos qualitativos ${ }^{(53)}$ e foi aplicada a regressão linear múltipla para o desfecho continuo tempo de internação hospitalar ${ }^{(54)}$.

\subsection{Aprovação pela Comissão de Ética}

O estudo foi aprovado pela Comissão de Ética e Pesquisa do ICrHCFMUSP e pela Comissão de Ética para Análise de Projetos de Pesquisa (Capes) em 14 de fevereiro de 2014 com Protocolo de pesquisa de número 1153/02/2014 (Anexo D). 
4 RESULTADOS 


\subsection{Análise descritiva}

No período de 01 de janeiro de 2007 a 31 de dezembro de 2013, foram admitidos 12.559 RN no Centro Neonatal do ICrHCFMUSP, dos quais 80 (6,4 para cada 1.000 nascidos vivos) foram diagnosticados com MMC ao nascimento. A incidência anual da doença no período de análise está apresentada na tabela 1.

Tabela 1 - Incidência de MMC entre os nascidos vivos no Centro Neonatal do ICrHCFMUSP por ano de estudo

\begin{tabular}{ccccc}
\hline $\begin{array}{c}\text { Ano de } \\
\text { estudo }\end{array}$ & $\begin{array}{c}\text { Total de } \\
\text { nascimentos }\end{array}$ & $\begin{array}{c}\text { Nascidos vivos } \\
\text { com MMC }\end{array}$ & $\begin{array}{c}\text { Incluidos } \\
\text { no estudo }\end{array}$ & $\begin{array}{c}\text { Incidência de MMC } \\
\text { (Casos/1.000NV) }\end{array}$ \\
\hline 2007 & 1928 & 15 & 13 & 7,8 \\
2008 & 1610 & 8 & 8 & 5,0 \\
2009 & 1726 & 14 & 11 & 8,1 \\
2010 & 1809 & 9 & 9 & 5,0 \\
2011 & 1875 & 11 & 8 & 5,9 \\
2012 & 1804 & 11 & 11 & 6,1 \\
2013 & 1807 & 12 & 10 & 6,6 \\
\hline Total & $\mathbf{1 2 5 5 9}$ & 80 & 70 & 6,4 \\
\hline
\end{tabular}


De acordo com os critérios de inclusão e exclusão, foram analisados 70 neonatos. Um paciente com MMC cervical e 9 pacientes com outras malformações e/ou síndromes genéticas foram excluídos da análise (Tabela 2).

Tabela 2 - Incidência de malformações e síndromes genéticas associadas à MMC em 9 neonatos excluídos do estudo

\begin{tabular}{lc}
\hline Malformações / Síndromes genéticas & Frequência \\
\hline Cardiopatia & $\mathbf{N}(\%)$ \\
Cromossomopatia & $4(5,0)$ \\
Onfalocele & $1(1,2)$ \\
Encefalocele & $3(3,7)$ \\
\hline Total & $1(1,2)$ \\
\hline
\end{tabular}

Quanto às variáveis relativas às gestantes, os dados são apresentados na tabela 3. A média das idades foi de 27 anos, sendo a maioria primigesta $(35,7 \%)$. As intercorrências de gestação foram infrequentes; o parto eletivo ocorreu em $96 \%$ dos casos e apenas três gestações foram interrompidas por sofrimento fetal. Em todos os casos a resolução da gestação foi por parto cesáreo. 
Tabela 3 - Estatística descritiva das características maternas

\begin{tabular}{lc}
\hline Variáveis Categóricas & Frequência No $(\%)$ \\
\hline № gestações anteriores & $25(35,7)$ \\
0 & $22(31,4)$ \\
1 & $23(32,9)$ \\
$\geq 2$ & \\
Tipo de parto & $0(0)$ \\
Normal & $0(0)$ \\
Forceps & $70(100)$ \\
Cesáreo & \\
Comorbidades Maternas & $2(2,9)$ \\
Epilepsia & $2(2,9)$ \\
Asma & $3(4,4)$ \\
Hipotireoidismo & $2(2,9)$ \\
DM & $0(0)$ \\
RPMO & $0(0)$ \\
TPP & $3(4,3)$ \\
SFA & $38,3(310)$ \\
DHEG & Mediana (Mínimo - Máximo $)$ \\
\hline Variáveis Contínuas & $26(15-43)$ \\
\hline Idade Materna (anos) & \\
IG (semanas) & \\
\hline
\end{tabular}

No que diz respeito aos $\mathrm{RN}$, os dados são apresentados na tabela 4 . MMC foi mais prevalente no gênero feminino, na proporção de $1,4: 1$ casos para o gênero feminino e masculino, respectivamente. A prematuridade 
ocorreu em apenas seis pacientes $(8,5 \%)$. O peso médio ao nascer foi de 3.024 gramas e, ao relacionar o peso com a idade gestacional de nascimento, $87,1 \%$ dos neonatos foram classificados como adequados para a idade gestacional (AIG).

Tabela 4 - Estatística descritiva das características dos RN

\begin{tabular}{lc}
\hline Variáveis Categóricas & Frequência No $(\%)^{\circ}$ \\
\hline Gênero feminino & $41(58,6)$ \\
Classificação gestacional & $8(11,4)$ \\
PIG & $61(87,1)$ \\
AIG & $1(1,4)$ \\
GIG & $6(8,6)$ \\
IG <37 semanas & Média / Mediana (Mínimo - Máximo) \\
\hline Variáveis Contínuas & 35,4 / $35(28$ - 50) \\
\hline PC (cm) & 3.024 / 3.065 (880 - 4.150) \\
PN (gramas)
\end{tabular}

As características anatômicas e cirúrgicas da MMC são apresentadas na tabelas 5 e 6 . Houve predomínio de lesões em topografia lombar e com perda da integridade das membranas que recobrem o defeito. A correção do defeito medular ocorreu nas primeiras 72 horas de vida em 90\% dos casos. Em 38\% deles a cirurgia foi imediata, ou seja, o paciente foi mantido no centro cirúrgico para o procedimento antes de ser transferido para a UTI neonatal. 
Tabela 5 - Estatística descritiva das características anatômicas da MMC

\begin{tabular}{lc}
\hline Variáveis Categóricas & Frequência $\mathbf{N}^{\circ}(\%)$ \\
\hline Topografia & $4(5,7)$ \\
Torácica & $59(84,3)$ \\
Lombar & $5(7,1)$ \\
Sacral & $2(2,8)$ \\
Não Classificada & $11(15,7)$ \\
Lesão extensa & $54(75,7)$ \\
HC antenatal & $11(15,7)$ \\
Macrocrania & $45(64,3)$ \\
Rotura de membranas que recobrem o defeito & Média / Mediana (Mínimo - Máximo) \\
\hline Variável Contínua & 35,4 / 35 (28 - 50) \\
\hline PC (cm) & \\
\hline
\end{tabular}

Tabela 6 - Estatística descritiva das características da correção cirúrgica da MMC

\begin{tabular}{lc}
\hline Variáveis Categóricas & Frequência No $(\%)^{\circ}$ \\
\hline Momento da correção cirúrgica & $46(65,7)$ \\
0 - 24h & $14(20)$ \\
25 - $48 \mathrm{~h}$ & $4(5,7)$ \\
49 - 72h & $6(8,6)$ \\
$>72 h$ & $27(38,6)$ \\
Correção cirúrgica imediata & $11(15,7)$ \\
Necessidade de retalho cirúrgico & Média / Mediana (Mínimo - Máximo) \\
\hline Variável Contínua & 26,0 / $8(1$ - 201) \\
\hline Correção Cirúrgica (horas)
\end{tabular}


$\mathrm{Na}$ tabela 7 são apresentados os dados do atendimento inicial em sala de parto. Foi necessária intervenção pediátrica com suporte ventilatório por meio de pressão positiva em $22,9 \%$ dos nascimentos. Nenhum paciente necessitou de compressões torácicas ou uso de adrenalina.

Tabela 7 - Estatística descritiva do atendimento em sala de parto

\begin{tabular}{lc}
\hline Variáveis categóricas & $\begin{array}{c}\text { Frequência } \\
\mathbf{N}^{\circ}(\%)\end{array}$ \\
\hline VPP & $16(22,9)$ \\
Massagem cardíaca e/ou uso de Adrenalina & $0(0)$ \\
Necessidade de intubação endotraqueal & $8(11,4)$ \\
Apgar $1^{\circ}$ minuto $\leq 3$ & $4(5,7)$ \\
Apgar $1^{\circ}$ minuto $<7$ & $11(15,7)$ \\
Apgar $5^{\circ}$ minuto $\leq 3$ & $0(0)$ \\
Apgar $5^{\circ}$ minuto $<7$ & $2(2,8)$ \\
\hline
\end{tabular}

O tempo médio de internação e a frequência absoluta e relativa de cada desfecho de curto prazo são apresentados na tabela 8. Houve três óbitos no período de análise (4,3\%). Um paciente faleceu com quatro dias de vida devido à pneumonia aspirativa. Outros dois óbitos foram secundários à sepse com infecção do SNC. Em todos os pacientes foi avaliada a necessidade de derivação ventricular sendo que 33 casos foram submetidos ao procedimento durante a internação hospitalar. Nove pacientes necessitaram de derivação ventricular externa (DVE), sendo dois neonatos para tratamento de fístula liquórica e 7 para tratamento de ventriculite associada à dilatação ventricular 
progressiva. As complicações infecciosas foram avaliadas separadamente e como desfecho combinado. O evento sepse e/ou infecção do SNC ocorreu em 24 pacientes $(34,9 \%)$.

Dos $11 \mathrm{RN}$ com diagnóstico de ventriculite, foi possível isolar o agente infeccioso no LCR em três casos. Acinetobacter baumannii, Klebisiella pneumoniae e Enterobacter cloacae foram identificados em um paciente cada. $\mathrm{RN}$ com ventriculite receberam empiricamente vancomicina associada à meropenem ou cefepime antes dos resultados da cultura do LCR. O tempo médio de antibiótico por via intravenosa foi de 23,6 dias. Entre os neonatos com ventriculite, o tempo médio de internação foi 2,5 vezes maior quando comparado com o tempo médio de internação de todos os pacientes incluídos no estudo, 69,3 dias vs. 28 dias, respectivamente.

Tabela 8 - Estatística descritiva dos desfechos de curto prazo analisados durante internação hospitalar

\begin{tabular}{lc}
\hline Variáveis Categóricas & ${\text { Frequência } \mathbf{N}^{\circ}(\%)}^{\circ}$ \\
\hline DVP & $32(45,7)$ \\
Revisão de DVP & $8(25)$ \\
DVE & $9(12,9)$ \\
Infecção de FO & $9(12,9)$ \\
Deiscência de FO & $27(38,6)$ \\
Sepse & $20(28,6)$ \\
Infecção de SNC & $11(15,7)$ \\
Óbito & $3(4,3)$ \\
\hline Variável Contínua & Média / Mediana (Mínimo - Máximo) \\
\hline Tempo de Internação Hospitalar (dias) & 27,7 / $21(4$ - 196) \\
\hline
\end{tabular}




\subsection{Análise Bivariada}

\section{Reanimação em sala de parto}

Dentre os marcadores prognósticos avaliados nessa população, a presença de macrocrania ao nascer e o tamanho do defeito medular estão associados a uma maior necessidade de suporte ventilatório em sala de parto. As análises bivariadas com seus respectivos intervalos de confiança são apresentadas na tabela 9 .

Doze pacientes apresentaram macrocrania ao nascer, dos quais 7 (58\%) necessitaram de pressão positiva para manter a ventilação pulmonar adequada; entre os 59 pacientes sem macrocrania, a necessidade de VPP foi menor, realizada em apenas $10 \mathrm{RN}(17 \%)$.

Quanto à necessidade de intubação endotraqueal, a macrocrania ao nascer esteve associada a um maior risco. O procedimento foi realizado em quatro $(33,3 \%)$ de 12 pacientes com macrocrania vs. quatro $(6,8 \%)$ de 59 pacientes com PC normal.

Os pacientes com maiores defeitos medulares receberam 3,2 vezes mais suporte ventilatório. Entre os 11 pacientes com lesão extensa, seis $(54,5 \%)$ foram ventilados com pressão positiva vs.10 (16,9\%) de 59 pacientes com defeito medular de menor extensão. A necessidade de intubação orotraqueal também foi maior. Cinco $(45,5 \%)$ de 11 pacientes com lesão extensa foram submetidos ao procedimento vs. cinco $(8,5 \%)$ de 59 pacientes com defeitos não extensos. 
Tabela 9 - Análise bivariada dos marcadores prognósticos vs. VPP e intubação endotraqueal

\begin{tabular}{lcccc}
\hline \multicolumn{1}{c}{ Marcador Prognóstico } & \multicolumn{2}{c}{ VPP } & \multicolumn{2}{c}{ Intubação Endotraqueal } \\
\hline & $\mathrm{p}$ & $\mathrm{RR}(\mathrm{IC} 95 \%)$ & $\mathrm{p}$ & $\mathrm{RR}(\mathrm{IC} 95 \%)$ \\
\cline { 2 - 5 } & 0,99 & $1,07(0,18-6,16)$ & 0,80 & $2,28(0,36-14,33)$ \\
$\begin{array}{l}\text { Topografia: } \\
\text { Torácica vs. Sacral/Lombar }\end{array}$ & 0,03 & $3,22(1,47-7,03)$ & 0,003 & $8,94(2,49-32,1)$ \\
$\begin{array}{l}\text { Tamanho: } \\
\text { Extensa vs. Não extensa }\end{array}$ & 0,09 & $4,81(0,68-33,78)$ & 0,74 & $2,24(0,29-16,97)$ \\
$\begin{array}{l}\text { HC antenatal: } \\
\text { Presente vs. Ausente }\end{array}$ & 0,03 & $3,22(1,47-7,03)$ & 0,03 & $5,36(1,57-18,3)$ \\
$\begin{array}{l}\text { Macrocrania ao nascer: } \\
\text { Presente vs. Ausente }\end{array}$ & 0,91 & $1,22(0,48-3,12)$ & 0,80 & $1,66(0,36-7,65)$ \\
$\begin{array}{l}\text { Membranas que recobrem o } \\
\text { defeito: Rotas vs. Íntegras }\end{array}$ & & & & \\
\hline
\end{tabular}

\section{Tempo de internação hospitalar}

As análises bivariadas dos marcadores prognósticos com o tempo de internação hospitalar são apresentadas na tabela 10. A presença de HC antenatal, a topografia da lesão e a extensão da MMC apresentaram associação estatística com hospitalização prolongada.

Tabela 10 - Análise bivariada dos marcadores prognósticos vs. tempo de internação hospitalar

\begin{tabular}{lcc}
\hline \multicolumn{1}{c}{ Marcador Prognóstico } & $\mathbf{p}$ & $\begin{array}{c}\text { Tempo de Internação } \\
\text { dias (mediana) }\end{array}$ \\
\hline Topografia lesão: Torácica vs. Sacral / Lombar & 0,03 & 49,5 vs. 20 \\
Tamanho lesão: Extensa vs. Não extensa & 0,008 & 48 vs. 20 \\
HC antenatal: Presente vs. Ausente & 0,01 & 23 vs. 10 \\
Macrocrania ao nascer: Presente vs. Ausente & 0,16 & 25 vs. 20 \\
Membranas que recobrem o defeito: Rotas vs. Íntegras & 0,23 & 22 vs. 16 \\
Correção cirúrgica: >48h vs. $\leq 48 \mathrm{~h}$ & 0,64 & 22,5 vs. 20,5 \\
Correção cirúrgica: Imediata vs. Não imediata & 0,65 & 23 vs. 20 \\
\hline
\end{tabular}




\section{Necessidade de derivação ventricular}

Quando o desfecho necessidade de derivação ventricular foi avaliado, apenas a presença de $\mathrm{HC}$ antenatal e a macrocrania ao nascer apresentaram associação significante.

Dez (83\%) de 12 pacientes com macrocrania vs. 23 (39\%) de 59 pacientes com PC normal foram submetidos à derivação ventricular. Trinta e um (57\%) dos 54 pacientes com HC foram derivados vs. três $(17,6 \%)$ de 17 pacientes sem HC. As análises bivariadas com seus respectivos intervalos de confiança são apresentadas na tabela 11.

Tabela 11 - Análise bivariada dos marcadores prognósticos vs. necessidade de derivação ventricular

\begin{tabular}{lcc}
\hline \multicolumn{1}{c}{ Marcador Prognóstico } & $\mathbf{p}$ & RR (IC 95\%) \\
\hline Topografia lesão: Torácica vs. Sacral / Lombar & 0,99 & $1,10(0,39-3,05)$ \\
Tamanho lesão: Extensa vs. Não extensa & 0,33 & $1,50(0,88-2,57)$ \\
HC antenatal: Presente vs. Ausente & 0,01 & $3,10(1,08-8,91)$ \\
Macrocrania ao nascer: Presente vs. Ausente & 0,02 & $2,09(1,37-3,21)$ \\
Membranas que recobrem o defeito: Rotas vs. Íntegras & 0,05 & $1,98(1,00-3,92)$ \\
Correção cirúrgica: >48h vs. s48h & 0,97 & $0,86(0,38-1,92)$ \\
Correção cirúrgica: Imediata vs. Não imediata & 0,99 & $1,05(0,62-1,78)$ \\
\hline
\end{tabular}

\section{Complicação relacionada à Ferida Operatória}

A extensão da $M M C$ foi associada à deiscência e infecção de FO. Oito de 11 pacientes (73\%) com lesão extensa apresentaram deiscência e 5 (45\%) pacientes foram diagnosticados com infecção local. Entre os 59 pacientes com lesão de menor extensão 19 (32,2\%) apresentaram deiscência 
da sutura e quatro desenvolveram infecção (6,7\%). As análises bivariadas são apresentadas na tabela 12 .

Tabela 12 - Análise bivariada dos marcadores prognósticos vs. infecção e deiscência de FO

\begin{tabular}{|c|c|c|c|c|}
\hline \multirow[t]{2}{*}{ Marcador Prognóstico } & \multicolumn{2}{|c|}{ Infecção de FO } & \multicolumn{2}{|c|}{ Deiscência de FO } \\
\hline & $\mathrm{p}$ & RR (IC 95\%) & $\mathrm{p}$ & RR (IC 95\%) \\
\hline $\begin{array}{l}\text { Topografia lesão: } \\
\text { Torácica vs. Sacral / Lombar }\end{array}$ & 0,88 & $2(0,32-12,3)$ & 0,99 & $1,33(0,47-3,73)$ \\
\hline $\begin{array}{l}\text { Tamanho lesão: } \\
\text { Extensa vs. Não extensa }\end{array}$ & 0,007 & $6,70(2,13-21,1)$ & 0,03 & $2,26(1,35-3,79)$ \\
\hline $\begin{array}{l}\text { HC antenatal: } \\
\text { Presente vs. Ausente }\end{array}$ & 0,99 & $1,12(0,26-4,89)$ & 0,24 & $1,84(0,74-4,58)$ \\
\hline $\begin{array}{l}\text { Macrocrania ao nascer: } \\
\text { Presente vs. Ausente }\end{array}$ & 0,29 & $2,68(0,78-9,15)$ & 0,99 & $0,93(0,40-2,17)$ \\
\hline $\begin{array}{l}\text { Membranas que recobrem o } \\
\text { defeito: Rotas vs. Íntegras }\end{array}$ & 0,61 & $1,94(0,44-8,66)$ & 0,56 & $1,32(0,68-2,57)$ \\
\hline $\begin{array}{l}\text { Correção cirúrgica: } \\
>48 \mathrm{~h} \text { vs. } \leq 48 \mathrm{~h}\end{array}$ & 0,99 & $0,75(0,10-5,37)$ & 0,64 & $1,36(0,67-2,75)$ \\
\hline $\begin{array}{l}\text { Correção cirúrgica: } \\
\text { Imediata vs. Não imediata }\end{array}$ & 0,45 & $0,50(0,15-1,71)$ & 0,29 & $0,67(0,38-1,21)$ \\
\hline
\end{tabular}

\section{Complicações infecciosas}

As análises bivariadas dos desfechos infecciosos com as variáveis de interesse estão apresentadas na tabela 13.

Infecção do SNC foi mais frequente nos pacientes com defeitos extensos de fechamento do tubo neural. O diagnóstico foi feito em cinco $(31,2 \%)$ de 11 pacientes com defeitos extensos vs. seis $(10,2 \%)$ de 59 pacientes com defeitos de menor extensão. 
Tabela 13 - Análise bivariada dos marcadores prognósticos vs. infecção de SNC e sepse

\begin{tabular}{|c|c|c|c|c|}
\hline \multirow[t]{2}{*}{ Marcador Prognóstico } & \multicolumn{2}{|c|}{ Infecção de SNC } & \multicolumn{2}{|r|}{ Sepse } \\
\hline & $P$ & $\mathrm{RR}(\mathrm{IC} 95 \%)$ & $p$ & $\mathrm{RR}(\mathrm{IC} 95 \%)$ \\
\hline $\begin{array}{l}\text { Topografia lesão: Torácica vs. } \\
\text { Sacral / Lombar }\end{array}$ & 0,24 & $3,56(1,12-11,25)$ & 0,13 & $3(1,48-6,08)$ \\
\hline $\begin{array}{l}\text { Tamanho lesão: Extensa vs. Não } \\
\text { extensa }\end{array}$ & 0,02 & $4,47(1,65-12,11)$ & 0,09 & $2,29(1,13-4,66)$ \\
\hline HC antenatal: Presente vs. Ausente & 0,38 & $3,21(0,44-23,27)$ & 0,41 & $1,81(0,60-5,45)$ \\
\hline $\begin{array}{l}\text { Macrocrania ao nascer: Presente vs. } \\
\text { Ausente }\end{array}$ & 0,99 & $1,19(0,29-4,78)$ & 0,09 & $2,29(1,13-4,66)$ \\
\hline $\begin{array}{l}\text { Membranas que recobrem o defeito: } \\
\text { Rota vs. Íntegra }\end{array}$ & 0,33 & $2,5(0,58-10,68)$ & 0,37 & $1,67(0,69-4,04)$ \\
\hline Correção cirúrgica: $>48 \mathrm{~h}$ vs. $\leq 48 \mathrm{~h}$ & 0,37 & $2,25(0,71-7,07)$ & 0,99 & $1,06(0,38-2,96)$ \\
\hline $\begin{array}{l}\text { Correção cirúrgica: Imediata vs. Não } \\
\text { imediata }\end{array}$ & 0,39 & $0,52(0,18-1,55)$ & 0,33 & $0,63(0,31-1,31)$ \\
\hline
\end{tabular}

\section{Marcadores de bom prognóstico}

Foram considerados de evolução favorável aqueles pacientes que no período da análise não necessitaram de suporte em sala de parto, não foram submetidos à derivação ventricular e não evoluíram com complicações infecciosas ou complicações relacionadas à FO. Dentre os fatores de interesse avaliados no estudo, a ausência de hidrocefalia e o PC normal ao nascimento foram identificados como marcadores de bom prognóstico. Entre os 54 pacientes com HC, $13(24,1 \%)$ apresentaram evolução favorável vs. 12 $(70,6 \%)$ de 17 pacientes sem HC. Todos os neonatos portadores de macrocrania apresentaram intercorrências, sendo essas observadas em 25 $(66,4 \%)$ de 59 pacientes com PC normal. O resultado da análise bivariada é apresentado na tabela 14 . 
Tabela 14 - Análise bivariada dos marcadores prognósticos vs. desfecho combinado de evolução favorável

\begin{tabular}{lcc}
\hline \multicolumn{1}{c}{ Marcador Prognóstico } & $\mathbf{p}$ & RR (IC 95\%) \\
\hline Topografia lesão: Sacral / Lombar vs. Torácica & 0,99 & $1,17(0,64-2,12)$ \\
Tamanho lesão: Não extensa vs. Extensa & 0,33 & $1,34(0,95-1,89)$ \\
HC antenatal: Ausente vs. Presente & 0,009 & $1,73(1,39-2,16)$ \\
Macrocrania ao nascer: Ausente vs. Presente & 0,006 & $1,74(1,39-2,16)$ \\
Membranas que recobrem o defeito: Íntegras vs. Rotas & 0,41 & $1,23(0,82-1,83)$ \\
Correção cirúrgica: $\leq 48 h$ vs. >48h & 0,50 & $1,5(0,73-3,07)$ \\
Correção cirúrgica: Imediata vs. Não imediata & 0,27 & $0,80(0,56-1,1)$ \\
\hline
\end{tabular}

\subsection{Análise Multivariada}

As análises multivariadas são apresentadas na tabela 15.

As lesões extensas foram relacionadas com infecção do SNC, com complicações de FO e com maior tempo de internação hospitalar. A topografia torácica do defeito medular foi associada a maior tempo de internação hospitalar. A correção cirúrgica após 48 horas de vida esteve associada a 5,72 vezes mais risco de infecção do SNC. A ausência de HC antenatal foi um marcador de bom prognóstico no modelo multivariado conforme já apresentado na análise bivariada. 
Tabela 15 - Análise multivariada dos marcadores prognósticos vs. desfechos de curto prazo nos pacientes com MMC

\begin{tabular}{|c|c|c|}
\hline Desfechos curto prazo / Marcadores Prognósticos & $\mathbf{p}$ & IRR (IC 95\%) \\
\hline \multicolumn{3}{|l|}{ Reanimação em sala de parto } \\
\hline Topografia lesão: Sacral / Lombar & 0,34 & $2,83(0,34-23,73)$ \\
\hline Tamanho lesão: Extensa & 0,08 & $2,79(0,87-8,94)$ \\
\hline Idade gestacional & 0,40 & $0,88(0,66-1,18)$ \\
\hline Macrocrania ao nascer: Presente & 0,14 & $2,46(0,75-8,09)$ \\
\hline Sofrimento fetal agudo: Presente & 0,65 & $1,72(0,16-18,05)$ \\
\hline \multicolumn{3}{|l|}{ Derivação ventrículo peritoneal } \\
\hline Topografia lesão: Sacral / Lombar & 0,90 & $1,10(0,24-4,94)$ \\
\hline Tamanho lesão: Extensa & 0,39 & $1,48(0,60-3,61)$ \\
\hline Correçãocirúrgica: >48h & 0,72 & $0,80(0,24-2,67)$ \\
\hline HC antenatal: Presente & 0,08 & $2,90(0,87-9,65)$ \\
\hline \multicolumn{3}{|l|}{ Infecção de FO } \\
\hline Topografia lesão: Sacral / Lombar & 0,90 & $1,15(0,13-9,80)$ \\
\hline Tamanho lesão: Extensa & 0,004 & $7,98(1,93-33,02)$ \\
\hline Correçãocirúrgica: >48h & 0,83 & $0,77(0,08-7,77)$ \\
\hline $\mathrm{HC}$ antenatal: Presente & 0,69 & $0,68(0,11-4,25)$ \\
\hline \multicolumn{3}{|l|}{ Deiscência de FO } \\
\hline Topografia lesão: Sacral / Lombar & 0,86 & $1,14(0,25-5,08)$ \\
\hline Tamanho lesão: Extensa & 0,04 & $2,51(1,05-6,02)$ \\
\hline Correção cirúrgica: >48h & 0,55 & $1,39(0,47-4,14)$ \\
\hline HC antenatal: Presente & 0,35 & $1,67(0,56-4,96)$ \\
\hline \multicolumn{3}{|l|}{ Infecção do sistema nervoso central } \\
\hline Topografia lesão: Sacral / Lombar & 0,55 & $0,59(0,11-3,24)$ \\
\hline Tamanho lesão: Extensa & 0,01 & $5,14(1,37-19,35)$ \\
\hline Correção cirúrgica: $>48 \mathrm{~h}$ & 0,03 & $5,72(1,14-28,58)$ \\
\hline Membranas que recobem o defeito: Rotas & 0,11 & $4,17(0,73-23,87)$ \\
\hline Idade gestacional & 0,93 & $1,01(0,75-1,37)$ \\
\hline \multicolumn{3}{|l|}{ Sepse neonatal } \\
\hline Topografia lesão: Sacral / Lombar & 0,31 & $0,49(0,12-1,97)$ \\
\hline Tamanho lesão: Extensa & 0,14 & $2,20(0,77-6,32)$ \\
\hline Correção cirúrgica: >48h & 0,92 & $0,92(0,18-4,64)$ \\
\hline Membranas que recobem o defeito: Rotas & 0,59 & $1,35(0,45-4,03)$ \\
\hline Idade gestacional & 0,97 & $1,01(0,74-1,37)$ \\
\hline \multicolumn{3}{|l|}{ Desfechos de bom prognóstico } \\
\hline Topografia lesão: Sacral / Lombar & 0,79 & $1,37(0,13-14,38)$ \\
\hline Tamanho lesão: Extensa & 0,24 & $0,30(0,04-2,27)$ \\
\hline Correção cirúrgica: >48h & 0,25 & $1,92(0,63-5,84)$ \\
\hline Membranas que recobem o defeito: Rotas & 0,84 & $1,09(0,45-2,65)$ \\
\hline Idade gestacional & 0,77 & $1,04(0,80-1,35)$ \\
\hline $\mathrm{HC}$ antenatal: Presente & 0,01 & $0,35(0,15-0,81)$ \\
\hline Desfechos curto prazo / Marcadores Prognósticos & $\mathbf{p}$ & $\beta($ IC 95\%) \\
\hline \multicolumn{3}{|l|}{ Tempo de internação hospitalar } \\
\hline Topografia lesão: Sacral / Lombar & 0,004 & $-39,89[-66,17-(-13,61)]$ \\
\hline Tamanho lesão: Extensa & $<0,001$ & $32,16(15,28-49,04)$ \\
\hline Correção cirúrgica: >48h & 0,76 & $-2,68(-20,54-15,18)$ \\
\hline Idade gestacional & 0,43 & $-1,52(-5,38-2,33)$ \\
\hline
\end{tabular}


5 DISCUSSÃO 
A MMC é uma anomalia congênita frequente em nosso meio. Nos sete anos do estudo foram diagnosticados 80 pacientes com MMC entre 12.559 nascidos no mesmo período, com incidência de 6,37 casos para cada 1.000 nascidos vivos. Embora tenhamos observado incidência de casos superior à relatada na literatura, a maioria dos autores apresenta casuísticas que incluem outros defeitos de fechamento do tubo neural. Portanto, esse fato deve ser levado em consideração na comparação da incidência da doença entre as diferentes populações.

Em 2005, Cunha et al descreveram 0,6 casos de espinha bífida para cada 1.000 nascimentos na região de Pelotas entre janeiro de 1990 a dezembro de $2003^{(21)}$. Na região do Vale do Paraíba, que compreende 35 municípios do estado de São Paulo, foi relatado 0,68 casos de espinha bífida para cada 1.000 nascidos vivos em estudo transversal referente aos nascimentos ocorridos no ano de 2004 ${ }^{(20)}$. Em estudo mais recente, publicado em 2012 por de Araujo et al, foi relatada a prevalência de 0,6 casos de MMC por 1.000 nascidos vivos no ano de 2004, com redução da prevalência para 0,2 casos por 1.000 nascidos vivos em 2005 no estado do Rio Grande do Norte ${ }^{(55)}$.

O Brasil está entre os países com as maiores prevalências de espinha bífida, com média de 1,14 casos por 1.000 nascidos vivos. Já países como França e Inglaterra apresentam prevalências 10 a 15 vezes menores, média de 0,08 e 0,1 casos para cada 1.000 nascidos vivos, respectivamente ${ }^{(2)}$. 
Entre os fatores responsáveis pela diferente distribuição populacional da doença incluem-se os fatores demográficos como gênero ${ }^{(56)}$, idade materna durante a gestação ${ }^{(57)}$, número de gestações anteriores ${ }^{(56)}$, condição socioeconômica ${ }^{(58)}$, nível de escolaridade materna ${ }^{(59)}$ e etnia ${ }^{(60)}$. Entre os fatores ambientais e patologias maternas destacam-se: suplementação periconcepcional de ácido fólico ${ }^{(58)}$, obesidade ${ }^{(61)}$ e hiperinsulinismo ${ }^{(62)}$.

Quanto às características demográficas da população estudada, houve predomínio de casos de MMC no gênero feminino (58,6\%), na proporção de 1.4:1 casos para o gênero feminino e masculino, respectivamente. $O$ acometimento preferencial de mulheres é descrito na literatura. Em estudo publicado em 2000 por Whitman et al, 57,7\% dos neonatos com MMC eram mulheres ${ }^{(56)}$. A diferença no desenvolvimento embrionário, a maior susceptibilidade a insultos teratogênicos e a maior taxa de abortamento espontâneo no gênero masculino são apresentadas como possíveis justificativas para a maior prevalência observada ${ }^{(63)}$.

A idade das gestantes variou entre 15 e 43 anos, com média de 27 anos. Vinte mulheres $(28,6 \%)$ apresentaram idade inferior a 19 anos ou superior a 35 anos. A distribuição etária encontrada é compatível com os dados publicados por Petrova et al em 2009, que analisaram os registros de fetos e RN com espinha bífida da população de Arkhangelskaja Oblast, na Rússia, entre 1995 e 2004. Nesta região,26\% das gestantes apresentaram idade inferior a 19 anos ou superior a 35 anos $^{(64)}$.

A elevada prevalência de casos de espinha bífida entre gestantes nos extremos de idade é demonstrada em metanálise publicada em 2005 por Vieira et al. Os autores sugerem uma correlação em forma de "U" entre idade 
materna e espinha bífida, sendo de maior risco os filhos de gestantes com idade inferior a 19 ou superior a 40 anos $^{(57)}$.

Além da idade materna, o número de gestações anteriores também está relacionado a defeitos de fechamento do tubo neural. No presente estudo $35,7 \%$ eram primigestas e $32,8 \%$ das mulheres apresentavam duas ou mais gestações anteriores. A distribuição das mulheres quanto ao número de gestações é semelhante àquela publicada por Whitman et al. Estes autores relatam predomínio de primigestas (36,8\%), porém com número significativo de gestantes com duas ou mais gestações anteriores (35\%). O risco de espinha bífida entre as mulheres com duas ou mais gestações foi 2,1 vezes maior que o apresentado pelas primigestas ${ }^{(56)}$.

Dentre os fatores ambientais, a suplementação de ácido fólico no período perinatal tem papel bem estabelecido na epidemiologia da doença. O ácido fólico atua no processo de multiplicação celular, com efeito direto sobre o fechamento do tubo neural e por mecanismo epigenético através da regulação da expressão de genes envolvidos nesse processo ${ }^{(14)}$. As políticas públicas de fortificação de alimentos com o micronutriente estão entre as possíveis justificativas para a menor prevalência observada em alguns países. Em 1998, a Food and Drug Administration (FDA) dos Estados Unidos determinou o enriquecimento de cereais com ácido fólico com redução no número de casos.

Em estudo publicado em 2005 por Willians et al, a prevalência de espinha bífida no Estados Unidos foi analisada em três períodos: pré fortificação de cereais com ácido fólico (janeiro de 1995 a dezembro de 1996), fortificação opcional (janeiro de 1997 a setembro de 1998) e fortificação 
obrigatória sob regulamentação do FDA (outubro de 1998 a dezembro de 2002). Houve redução de 19 a $36 \%$ no número de casos entre os períodos do estudo ${ }^{(65)}$.

No Brasil, a fortificação de farinha com ferro e ácido fólico foi regulamentada em 2002 pela Resolução da Diretoria Colegiada n 344 que determinou adição de $0,15 \mathrm{mg}$ de ácido fólico para cada 10 gramas de farinha de trigo e milho. O efeito da medida sobre a prevalência de espinha bífida foi avaliado por Orioli et al, que verificaram redução de $40 \%$ na prevalência da doença entre os anos de 2004 e 2006, em 27 Estados do Brasil( ${ }^{(66)}$.

Conforme os dados demográficos da população avaliada por nós (Tabela 3 e 4) observamos que suas características são semelhantes àquelas dos indivíduos incluídos nos estudos supracitados. A maior incidência da doença em nossa casuística pode ser justificada, em parte, por se tratar de um hospital terciário e de referência nacional para gestações de alto risco, incluindo malformações como os defeitos de fechamento do tubo neural.

Aspectos éticos e legais também interferem na incidência da doença. É esperado um maior número de nascidos malformados em países em que a legislação não permite a interrupção da gestação, como é o caso do Brasil em relação à MMC. A característica retrospectiva do estudo impossibilitou a análise dos fatores ambientais envolvidos no desenvolvimento da doença. O perfil de consumo de ácido fólico foi um dos fatores não analisados.

$\mathrm{Na}$ Identificação de marcadores prognósticos na população estudada analisaremos inicialmente a topografia do defeito medular.

O defeito medular foi classificado quanto a sua topografia em lesão sacral, lombar ou torácica, sendo o padrão de distribuição encontrado similar àquele 
descrito na literatura. A região lombar foi a preferencialmente acometida com 59 casos (84,3\%). Em estudo das características clínicas e cirúrgicas da MMC, realizado por Bulbul et al em 2010, 93\% dos pacientes foram diagnosticados com lesão em topografia lombar vs. $7 \%$ de casos com acometimento torácico ${ }^{(67)}$. Achado similar foi descrito por Tulipan et al em 2003, que observaram predomínio de MMC em topografia lombo sacral $(81 \%)^{(68)}$.

A topografia do defeito medular tem relevância clínica na avaliação desses pacientes. O comprometimento da medula e das raízes nervosas é maior quanto mais alto é o nível da lesão, sendo este um fator determinante no prognóstico neurológico. O nível da lesão medular influencia o desempenho escolar, comportamental e intelectual no longo prazo.

Em estudo publicado em 2005, Fletcher et al avaliaram 268 crianças portadoras de MMC entre 7 e 16 anos e encontraram associação entre o nível da lesão e desfechos neurocomportamentais ${ }^{(69)}$. Os autores avaliaram as crianças quanto à inteligência verbal e não verbal, leitura básica, compreensão de texto, desempenho em matemática e comportamento adaptativo. Entre os pacientes estudados, aqueles com lesão torácica apresentaram o pior desempenho. Quanto aos fatores que poderiam interferir no resultado, como intercorrências gestacionais e complicações relacionadas à derivação ventricular, o autor não identificou diferenças significativas entre os grupos. Contudo, outros fatores que poderiam contribuir para um pior prognóstico não foram avaliados, como ventriculomegalia ao nascer e ventriculite no período neonatal.

$\mathrm{Na}$ avaliação a curto prazo apresentada em nossa casuística, os pacientes com lesão em topografia torácica não apresentaram maior 
necessidade de derivação ventricular ou maior risco de complicações infecciosas. No entanto, o tempo médio de internação foi 2,5 vezes superior àquele dos pacientes com defeito em topografia sacral ou lombar $(p=0,004)$.

Em 2003 Rintoul et al analisaram a necessidade de derivação ventricular quanto à topografia do defeito medular em estudo retrospectivo de 297 pacientes. Diferente dos nossos achados, a necessidade de derivação ventricular foi mais frequente nos pacientes com lesão torácica. O procedimento foi realizado em $97 \%$ desses pacientes. Já os pacientes com lesão lombar e sacral necessitaram de derivação em $87 \%$ e $37 \%$ dos casos, respectivamente ${ }^{(31)}$. Segundo os autores, a maior parte das derivações ventriculares ocorreu na primeira semana de vida, sendo a mediana da idade para inserção de derivação ventrículo peritoneal igual a cinco dias.

Apesar de não identificarmos maior necessidade de derivação ventricular entre os pacientes com lesão em topografia torácica, esse resultado se refere ao período de seguimento, que se limitou ao momento da alta hospitalar. A mediana do tempo de seguimento desses $\mathrm{RN}$ foi de 21 dias, portanto superior à mediana da idade para derivação ventricular apresentada por Rintoul et $\mathrm{al}^{(31)}$. Esse comportamento sugere uma menor necessidade de derivação ventricular entre os pacientes da presente casuística.

Dentre os marcadores prognósticos, a extensão do defeito medular foi aquela que esteve mais frequentemente associado à evolução desfavorável. Os pacientes com os maiores defeitos medulares apresentaram 5,1 vezes mais infecção do SNC $(p=0,01)$. Apresentaram 7,9 vezes mais infecção de FO ( $p=0,004)$ e a internação hospitalar foi, em média, 32,2 dias mais longa $(p<0,001)$. 
A incidência de infecção de SNC observada em nosso estudo $(15,7 \%)$ é superior àquela observada por Charney et al (7\%) em estudo envolvendo 186 $\mathrm{RN}$ com $\mathrm{MMC}$ nos Estados Unidos ${ }^{(70)}$. Porém, é inferior à incidência verificada em outros países em desenvolvimento. Em estudo prospectivo realizado na Turquia entre janeiro de 2006 e dezembro de 2008, a incidência de infecção de SNC foi de $25 \%{ }^{(67)}$.

Em outro estudo, Demir et al avaliaram os fatores de risco para infecção e a eficácia da profilaxia com antibióticos nos pacientes com MMC. A incidência de infecção de SNC descrita $(19,8 \%)$ é superior àquela observada por nós ${ }^{(71)}$. Demir et al estratificaram os pacientes quanto ao tamanho da lesão em três grupos: defeitos menores que $26 \mathrm{~cm}^{2}$, defeitos com área entre $26 \mathrm{~cm}^{2}$ e $50 \mathrm{~cm}^{2}$ e defeitos maiores que $50 \mathrm{~cm}^{2}$. Foram comparadas as incidências de infecção SNC e infecção de FO entre eles.

Em concordância com os nossos achados, os pacientes com maiores defeitos apresentaram maior risco de infecção de FO. No total, $16(17,6 \%)$ de 91 pacientes avaliados foram diagnosticados com infecção de FO. Contudo, o autor não encontrou associação entre infecção do SNC e tamanho da lesão medular. Na nossa população o risco de infecção SNC foi 5,14 vezes maior entre os pacientes com defeitos extensos.

Os pacientes com MMC são submetidos mais frequentemente à VPP e à intubação endotraqueal durante atendimento inicial em sala de parto. A maior necessidade de suporte ventilatório pode ser justificada pelo controle central anormal da respiração ou pela paralisia de corda vocal ${ }^{(72)}$. Em estudo publicado por da Silva et al em 2013, a necessidade de VPP foi 4,5 vezes maior entre os 
pacientes com MMC. Dos 94 pacientes avaliados 29 necessitaram o procedimento (31\%), sendo que $20 \%$ deles apresentaram Apgar de 1 minuto menor que sete $e^{(73)}$.

Na população do nosso estudo $16(22,8 \%)$ RN necessitaram de VPP e $11(15,7 \%)$ apresentaram escore de Apgar de 1 minuto menor que 7, em concordância com da Silva et al. Na análise bivariada, os pacientes com maior extensão do defeito medular apresentaram necessidade 3,2 vezes maior de VPP $(p=0,03)$ e 8,9 vezes maior de intubação endotraqueal $(p=0,003)$ em relação aqueles com defeitos não extensos (Tabela 9). Não identificamos na literatura consultada por nós estudos comparando a necessidade de reanimação quanto à extensão do defeito medular.

Na MMC as alterações anatômicas do SNC impactam na dinâmica do fluxo liquórico com o surgimento da HC. No nível da fossa posterior, o deslocamento caudal da medula através do forame magno obstrui a drenagem do quarto ventrículo e compromete a circulação liquórica na junção cervicomedular. A HC também resulta de mecanismos não obstrutivos. A hipoplasia da fossa posterior presente na maioria desses pacientes impõe resistência à circulação cerebral, com aumento da pressão venosa e consequente perda da capacidade de reabsorção liquórica ${ }^{(74)}$.

A maior parte dos pacientes com MMC é diagnosticada com $\mathrm{HC}$ no período antenatal. Em estudo realizado por Babcook et al, 94\% dos fetos avaliados por exame ultrassonográfico com 24 semanas ou mais de gestação apresentavam ventriculomegalia ${ }^{(75)}$. Ao nascer, contudo, apenas um em cada 8 
RN apresenta PC acima do percentil 98 e somente 16\% manifestam clínica de hipertensão intracraniana ${ }^{(76)}$.

Os achados observados em nosso estudo foram semelhantes aos relatados por Babcook et al. Constatamos que $54(75,7 \%)$ neonatos apresentaram HC no período antenatal e 11 (20,4\%) foram diagnosticados com macrocrania ao nascer. $\mathrm{Na}$ análise bivariada, aqueles com macrocrania ao nascer necessitaram 3,2 vezes mais de $\operatorname{VPP}(p=0,03)$ e 5,4 vezes mais de intubação endotraqueal $(p=0,03)$ em relação aos neonatos com PC normal (Tabela 9). A presença de $\mathrm{HC}$ sem macrocrania não foi associada à maior intervenção em sala de parto $(p=0,09)$.

Embora não tenha sido encontrada associação estatisticamente significante entre macrocrania ao nascer e necessidade de reanimação em sala de parto na análise multivariada consideramos importante valorizar os resultados do modelo bivariado, pois tal achado interfere com as condutas assistenciais prestadas ao RN. É provável que os neonatos com macrocrania estejam no espectro de maior gravidade da doença, com maior comprometimento do fluxo liquórico e consequente dilatação ventricular progressiva. $O$ efeito pressórico do sistema supraventricular sobre as estruturas da fossa posterior pode justificar as alterações respiratórias observadas ao nascer, como apneia e obstrução de via aérea, sendo necessário maior suporte ventilatório. A maior necessidade de intervenção em sala de parto é um reconhecimento de gravidade e impõe que o atendimento em sala de parto seja feito por profissionais treinados em procedimentos de reanimação e com experiência em via aérea difícil. 
Em contrapartida, a ausência de $\mathrm{HC}$ representa o espectro de menor gravidade da doença. Entre os pacientes avaliados, aqueles sem HC antenatal apresentaram 1,7 vezes menos intercorrências no curto prazo como complicações com a FO, complicações infecciosas, necessidade de derivação ventricular e necessidade de reanimação em sala de parto $(p=0,01)$. Constatamos, portanto, que a ausência de HC no exame ultrassonográfico antenatal pode ser utilizada como marcador de bom prognóstico.

A MMC pode ser classificada quanto ao aspecto anatômico de apresentação. A lesão pode ser plana e displásica, com saída de líquido cefalorraquidiano (LCR) do espaço dural, ou do tipo cística e fechada, com formação de um saco herniário contendo meninges, tecido medular e LCR que se projeta através do defeito da coluna vertebral. A rotura das membranas pode ocorrer intra-útero, no momento do parto ou na vida pós-natal. A solução de continuidade imposta pela presença da fístula líquoríca pode estar implicada em maior risco infeccioso.

No nosso estudo, $45(64,3 \%)$ pacientes apresentaram membranas rotas no nascimento. A frequência encontrada é maior que a relatada na literatura. Em estudo realizado por Pinto et al em 2009 envolvendo RN com MMC submetidos a correção neurocirúrgica pós natal, a presença de fístula liquórica foi diagnosticada em $54,5 \%$ dos pacientes ${ }^{(45)}$.

Cuppen et al, em 2011, analisaram a influência do tipo de parto nos desfechos neurológicos dos pacientes com MMC submetidos à correção cirúrgica pós-natal. A presença de fistula liquórica foi relatada em 35,3\% dos pacientes e não houve diferença estatística quanto à via de parto adotada ${ }^{(77)}$. 
Em estudo realizado por Wilson et al, em 2007, comparando o prognóstico funcional de membros inferiores em pacientes com MMC, a correção cirúrgica foi realizada intra-útero entre 20 e 25 semanas de gestação. Nessa casuística 70\% dos pacientes apresentaram saco herniário íntegro no momento da correção cirúrgica ${ }^{(78)}$.

Apesar do potencial risco infeccioso determinado pela presença de fístula liquórica, os pacientes com membranas rotas por nós analisados não apresentaram maiores taxas de infecção de SNC ou sepse. Da mesma forma, não houve maior incidência de complicações de FO (Tabela 15). Em contrapartida, no estudo retrospectivo de $91 \mathrm{RN}$ portadores de $\mathrm{MMC}$ publicado em 2015 por Demir et al, as lesões medulares sem a cobertura de membranas apresentaram 8,6 vezes mais risco de meningite em relação àquelas com membranas íntegras ${ }^{(71)}$.

No presente estudo a correção cirúrgica ocorreu nas primeiras 48 horas na maioria dos pacientes com membranas rotas $(91,1 \%)$ sendo que $65 \%$ dos casos foram operados nas primeiras 24 horas. Já no estudo de Demir et al a correção cirúrgica nas primeiras 24 horas ocorreu em $36 \%$ dos pacientes. A menor exposição do defeito medular ao ambiente e o uso de antibiótico profilático precoce podem ter evitado a contaminação do SNC nos pacientes com perda de integridade de membranas da nossa casuística.

O tratamento cirúrgico da mielomenigocele ocorreu na maioria dos casos nas primeiras 48 horas de vida. Apenas $10(14,3 \%)$ pacientes foram submetidos à correção cirúrgica após esse período. A mediana da idade dos pacientes quando operados foi de 8 horas (Tabela 5). A correção precoce foi 
protetora para infecção do SNC, esses pacientes apresentaram risco 5,7 vezes menor de apresentar ventriculite $(p=0,03)$.

A correção cirúrgica imediatamente após o nascimento foi realizada em 27 pacientes (38,6\%). Sua vantagem teórica é a menor exposição do tecido medular ao ambiente, com menor incidência de distúrbio hidroeletrolítico por perda liquórica e menor risco de ruptura de membranas que envolvem o defeito. Em estudo caso controle publicado por Pinto et al em 2009, a correção cirúrgica imediata foi comparada ao controle histórico de correção cirúrgica pós-natal. Os autores demonstraram benefícios do procedimento em "tempo zero" com menor incidência de deiscência de FO e menor incidência de atraso do neurodesenvolvimento com um ano de vida ${ }^{(45)}$. Contudo, na nossa população, os pacientes submetidos ao procedimento em "tempo zero" não mostraram proteção adicional quanto a infecção de SNC (Tabela 13).

Em estudo prospectivo realizado por Bulbul et al em 2010, 28 pacientes com MMC foram comparados quanto aos desfechos de curto prazo e o efeito da correção cirúrgica precoce na morbimortalidade neonatal ${ }^{(67)}$. Entre os pacientes submetidos ao procedimento após o terceiro dia de vida a incidência de infecção do SNC foi maior, assim como a necessidade de uso de antibióticos e o tempo de internação hospitalar.

Em outro estudo retrospectivo realizado na Turquia entre 2009 e 2011 por Oncel et al foi avaliado o papel do momento da correção cirúrgica na evolução de curto prazo desses pacientes ${ }^{(79)}$. Nesse estudo a correção cirúrgica ocorreu mais tardiamente, em média com 8,2 dias de vida. 
Nos pacientes operados antes de cinco dias de vida o tempo de internação e a necessidade de antibióticos foi menor.

Pelo exposto, o estudo demonstrou elevada incidência de MMC na população analisada. O perfil demográfico desses pacientes contribuiu para esse achado. A análise das características anatômicas do defeito medular e da sua correção neurocirúrgica permitiu identificar marcadores prognósticos de curto prazo. 
6 CONCLUSÕES 
O estudo dos marcadores prognósticos em recém-nascidos portadores de Mielomeningocele nos permitiu chegar às seguintes conclusões:

- No período de sete anos foram diagnosticados 80 pacientes com MMC entre 12.559 nascidos vivos no Centro Neonatal do ICrHCFMUSP. A incidência foi de 6,4 casos para cada 1.000 nascidos vivos.

- Foram identificados os seguintes marcadores prognósticos para evolução desfavorável entre esses RN: Presença de macrocrania ao nascer, MMC extensa, MMC em topografia torácica.

- A presença de macrocrania ao nascer esteve associada à maior necessidade de reanimação em sala de parto em relação aos pacientes com PC normal. A necessidade de VPP foi 3,2 vezes maior e a necessidade de intubação endotraqueal foi 5,4 vezes maior nesses neonatos.

- O tamanho do defeito medular apresentou associação significativa com os desfechos infecciosos e com o tempo de internação hospitalar. Nos pacientes com lesão extensa a infecção de SNC foi 5,1 vezes mais frequente, a infecção de FO foi 7,9 vezes mais frequente e a internação hospitalar foi 32,2 dias mais longa. 
- Quanto à topografia da lesão, os pacientes com defeito torácico apresentaram tempo médio de internação hospitalar 2,5 vezes maior em relação àqueles com MMC sacral ou lombar.

- Foram identificados os seguintes marcadores prognósticos para evolução favorável em RN com MMC: Ausência de hidrocefalia antenatal e correção cirúrgica precoce da MMC.

- A ausência de HC constituiu um marcador de bom prognóstico, esses pacientes apresentaram um menor número de complicações durante a internação.

- A correção cirúrgica da MMC nas primeiras 48 horas de vida exerceu efeito protetor para infecção do SNC. Neonatos operados precocemente apresentaram 5,7 vezes menos ventriculite.

- A Integridade das membranas que recobrem o defeito medular e a correção cirúrgica em "tempo zero" não mostraram efeito protetor quanto aos desfechos pesquisados. 


\section{ANEXOS}




\section{Anexo A - Ficha de coleta dos dados}

\section{Dados Maternos: \\ Nome da mãe: \\ Idade (anos): \\ $\mathrm{RH}:$ \\ № gestações/№ partos: \\ Tipo de parto (Normal/Fórceps/Cesáreo): \\ Comorbidades Maternas \\ (Epilepsia/Asma/Hipotireoidismo/DM/RPMO/TPP/ SFA/DHEG):}

\section{Dados RN:}

DN:

Gênero (M/F):

IG (semanas):

PN (gramas):

$\mathrm{PC}(\mathrm{cm})$ :

$\operatorname{APGAR}\left(1^{\circ} \mathrm{min} / 5^{\circ} \mathrm{min}\right)$ :

Atendimento em sala de parto

(VPP/IOT/Compressão torácica/Uso de adrenalina):
Continuação...

3. Características Anatômicas MMC e do Tratamento cirúrgico:

Topografia (Torácica/Lombar/Sacral/Não classificada):

$\mathrm{HC}$ antenatal $(\mathrm{S} / \mathrm{N})$ :

Rotura das membranas que recobrem o defeito $(\mathrm{S} / \mathrm{N})$ :

Correção cirúrgica imediata $(\mathrm{S} / \mathrm{N})$ :

Momento da correção cirúrgica (horas):

Retalho cirúrgico $(\mathrm{S} / \mathrm{N})$ :

\section{Desfechos de curto prazo:}

$\operatorname{DVP}(\mathrm{S} / \mathrm{N})$ :

DVE $(\mathrm{S} / \mathrm{N})$ :

Revisão DVP (S/N):

Infecção FO (S/N):

Sepse $(\mathrm{S} / \mathrm{N})$ :

Infecção SNC (S/N):

Óbito (S/N):

Tempo Internação (dias):

\section{Observações:}

6. Data do levantamento do prontuário: 


\section{Anexo B - Curva de crescimento de Fenton para meninas ${ }^{(49)}$}

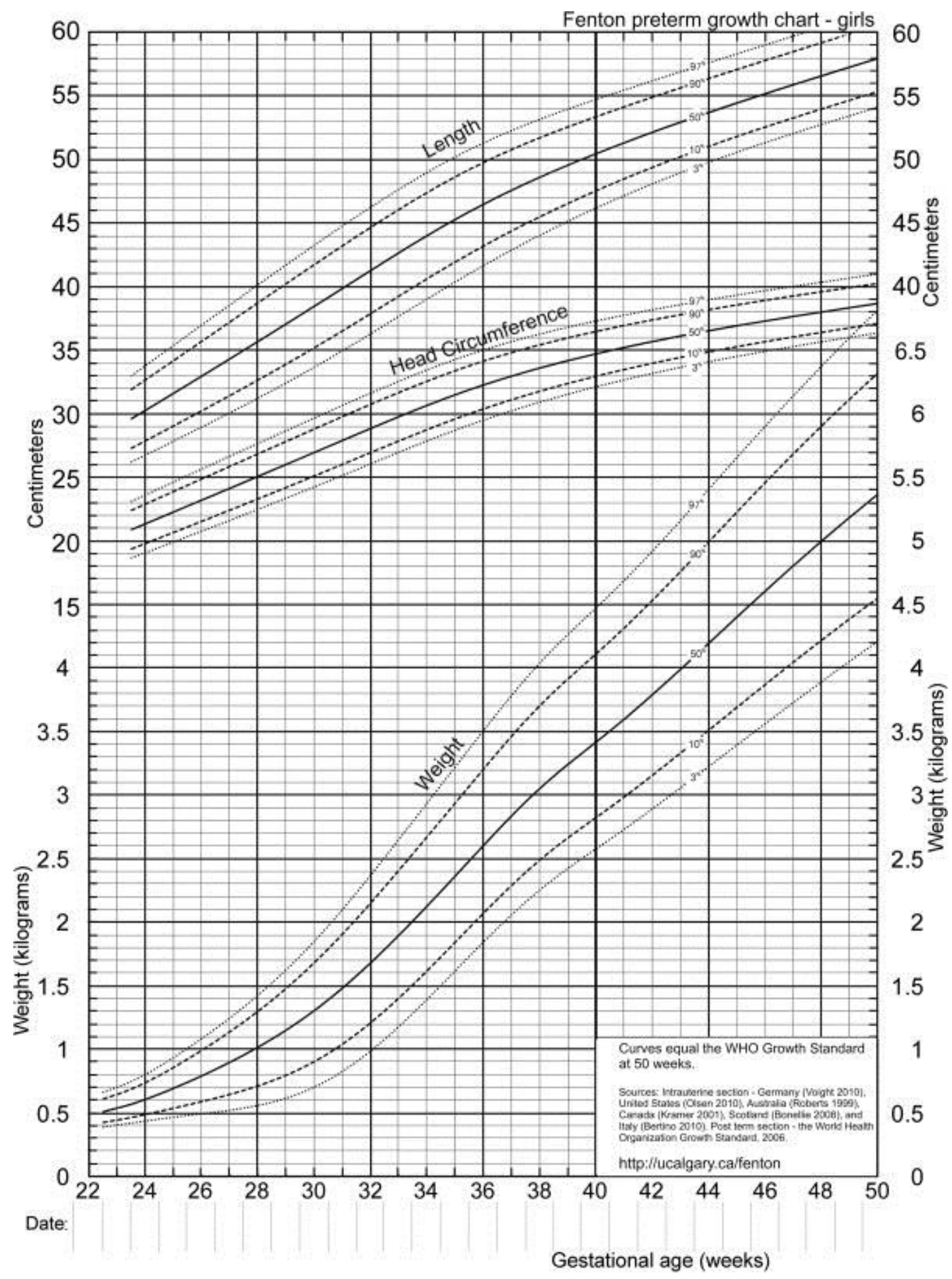




\section{Anexo C - Curva de crescimento de Fenton para meninos ${ }^{(49)}$}

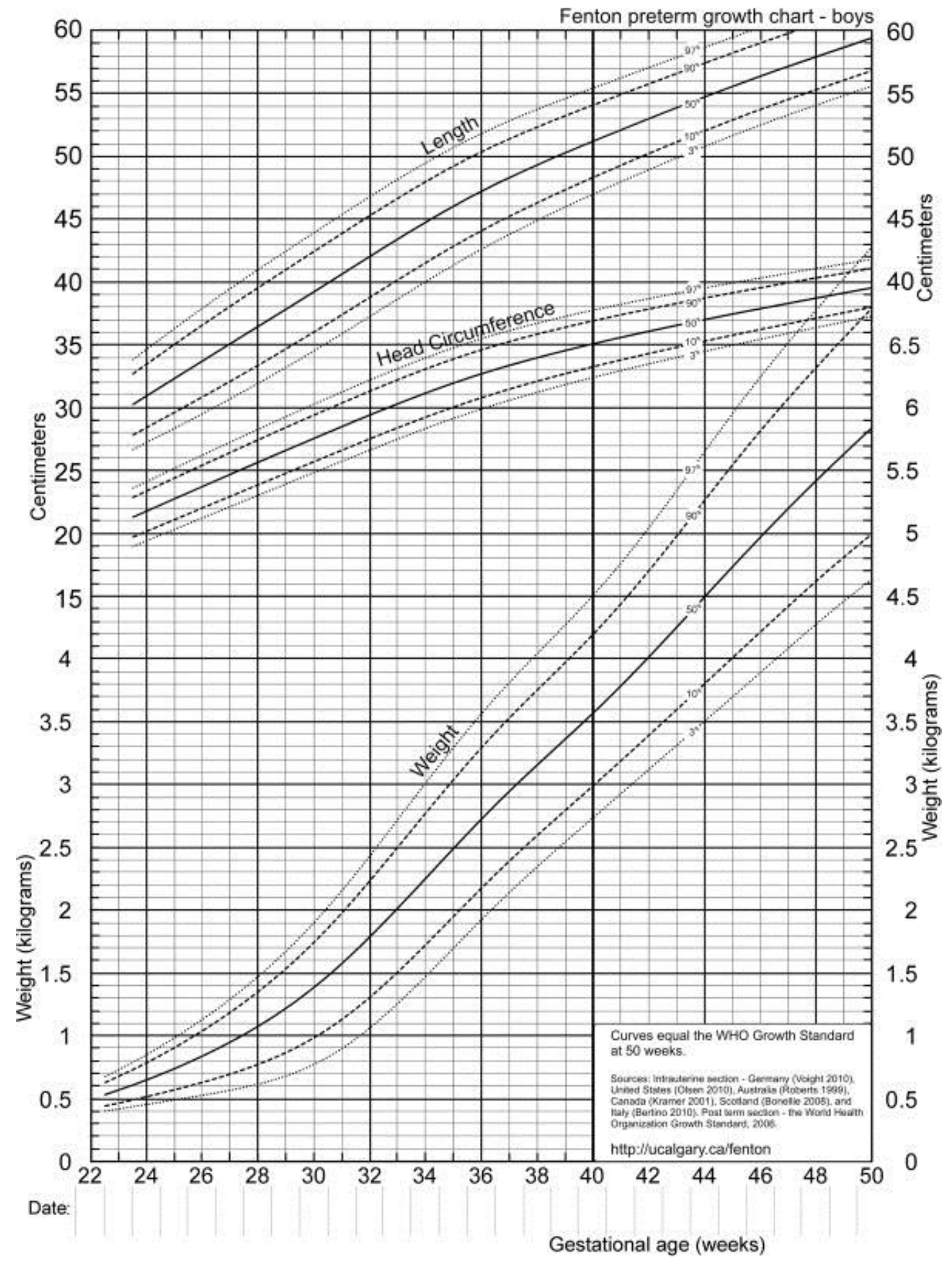




\title{
Anexo D - Parecer da Comissão de Ética para Análise de Projetos de Pesquisa - CAPPesq
}

\author{
Hospital das Clínicas da FMUSP \\ 1153 \\ Comissão de Ética para Análise de Projetos de Pesquisa - CAPPesq
}

\section{PROJETO DE PESQUISA}

Titulo: MARCADORES PROGNÓSTICOS EM RECÉM-NASCIDOS PORTADORES DE MIELOMENINGOCELE

Pesquisador Responsável: Vera Lucia Jornada Krebs

Versão: 1

Pesquisador Executante: Andre Broggin Dutra Rodrigues

CAAE: 27921114.7 .0000 .0068

Co-autores: Fernando Campos Gomes Pinto, Werther Brunow de Carvalho

Finalidade Académica Mestrado

Orientador: Vera Lucia Jornada Krebs

Instltuição: HCFMUSP

Departamento: PEDIATRIA

\section{PARECER CONSUBSTANCIADO DO CEP}

Registro on-line: 11803

Número do Parecer: 622.720

Data da Relatoria: 23/04/2014

Apresentação do Projeto: Trata-se de estudo retrospectivo, observacional, de consulta a prontuários de pacientes com diagnóstico de mielomeningocele para anotar alguns dados clinicos da evoluçăo dos mesmos.

Objetivo da Pesquisa: Visando descrever a incidência da patologia entre os neonatos com evolução desfavorável e analisar os fatores de risco para desfechos desfavoráveis a curto prazo desses pacientes.

Avaliação dos Riscos e Beneficios: Sem riscos.

Comentários e Considerações sobre a Pesquisa: Esta pesquisa clinica é um projeto de Dissertaçăo de Mestrado. Está bem descrita, tem numero importante de casos e pode servir eventualmente para orientar futuramente condutas nos pacientes com tal patologia.

Considerações sobre os Termos de apresentação obrigatória: Não se aplica.

Recomendaçōes: Sem recomendações particulares

Conclusōes ou Pendências e Lista de Inadequaçð̄es: Nada a destacar

Situação do Parecer: Aprovado

Necessita Apreciação da CONEP: Não

Considerações Finais a critério do CEP: Em conformidade com a Resolução CNS no 466/12 cabe ao pesquisador: a) desenvolver o projeto conforme delineado; b) elaborar e apresentar

Rua Dr. Ovidio Pires de Campos, 225 - Prédio da Administração - 59 andar CEP 05403-010 - São Paulo - SP.

5511 2661-7585 - 5511 2661-6442 ramais: 16, 17, 18 | marcia.carvalho@hc.fm.usp.br 


\section{Anexo D - Parecer da Comissão de Ética para Análise de Projetos de Pesquisa - CAPPesq}

\section{Hospital das Clínicas da FMUSP}

Comissão de Ética para Análise de Projetos de Pesquisa - CAPPesq

relatórios parciais e final; c)apresentar dados solicitados pelo CEP, a qualquer momento; d) manter em arquivo sob sua guarda, por 5 anos da pesquisa, contendo fichas individuais e todos os demais documentos recomendados pelo CEP; e) encaminhar os resultados para publicação, com os devidos créditos aos pesquisadores associados e ao pessoal técnico participante đo projeto; f) justificar perante ao CEP interrupção do projeto ou a não publicação dos resultados.

São Paulo, 25 de Abril de 2014

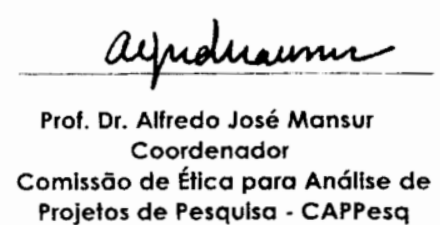


Anexo E - Artigo científico publicado pela revista Child's Nervous System com título "Short-term prognostic factors in myelomeningocele patients"

Short-term prognostic factors in myelomeningocele patients

Andre Broggin Dutra Rodrigues, Vera Lucia Jornada Krebs, Hamilton Matushita \& Werther Brunow de Carvalho

Child's Nervous System ISSN 0256-7040

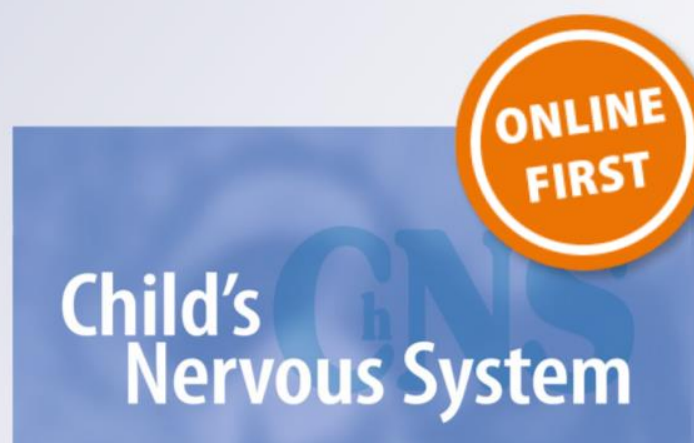

Official Journal of the

International Society

for Pediatric Neurosurgery

European Society

for Paediatric Neurosurgery

Korean Society

for Pediatric Neurosurgery

Japanese Society

for Pediatric Neurosurgery

Brazilian Society

for Pediatric Neurosurgery

Chinese Society

for Pediatric Neurosurgery

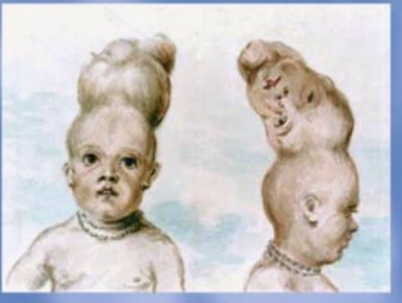

黑 Springer

算 Springer 
Your article is protected by copyright and all rights are held exclusively by SpringerVerlag Berlin Heidelberg. This e-offprint is for personal use only and shall not be selfarchived in electronic repositories. If you wish to self-archive your article, please use the accepted manuscript version for posting on your own website. You may further deposit the accepted manuscript version in any repository, provided it is only made publicly available 12 months after official publication or later and provided acknowledgement is given to the original source of publication and a link is inserted to the published article on Springer's website. The link must be accompanied by the following text: "The final publication is available at link.springer.com".

\section{Springer}




\section{Short-term prognostic factors in myelomeningocele patients}

Andre Broggin Dutra Rodrigues ${ }^{1,3}$ (C) Vera Lucia Jornada Krebs ${ }^{1}$. Hamilton Matushita $^{2} \cdot$ Werther Brunow de Carvalho ${ }^{1}$

Received: 4 November 2015 / Accepted: 4 January 2016

(C) Springer-Verlag Berlin Heidelberg 2016

\begin{abstract}
Purpose Patients with myelomeningocele have a high mortality and neurological disabilities that are correlated with the anatomical characteristics of the defect and with the development of acquired complications. The challenge in the postnatal management of myelomeningocele (MMC) is the early recognition of cases at risk for complications in order to establish individualized treatment strategies. This study aims to identify short-term prognostic markers for newborns with MMC. Anatomical characteristics of the spinal defect and technical aspects of the neurosurgical correction were analyzed for this purpose.

Methods A retrospective cohort study was conducted in 70 patients with MMC born between January 2007 and December 2013. Features of MMC anatomy and neurosurgical treatment were analyzed for the following outcomes: neonatal resuscitation, length of hospital stay, need for ventricular shunt, wound dehiscence, wound infection, central nervous system infection, and sepsis.

Results Large MMC was associated with central nervous system (CNS) infection, wound complications, and longer hospital stay. Patients with thoracic MMC required longer hospital stay. Surgical repair performed after $48 \mathrm{~h}$ of life increased in

Andre Broggin Dutra Rodrigues

andre@clinicadutrarodrigues.com.br

Discipline of Pediatrics, Hospital das Clínicas, São Paulo University Medical School, São Paulo, Brazil

2 Division of Neurosurgery, Hospital das Clínicas, São Paulo University Medical School, São Paulo, Brazil

3 Alameda Joaquim Eugênio de Lima, 881 — Jardim Paulista, São Paulo, SPConjunto 101, 01403-001, Brazil
\end{abstract}

5.72 times the risk of CNS infection. Absence of antenatal hydrocephalus was a favorable prognostic marker.

Conclusion Extent of the spinal cord defect and the time of surgical correction influenced the short-term outcomes of patients with myelomeningocele. Extensive lesions were associated with higher rates of CNS infections, surgical wound complications, and prolonged hospital stay. Interventions performed within $48 \mathrm{~h}$ after birth significantly reduced occurrence of CNS infections. Absence of antenatal hydrocephalus was associated with fewer complications in the first days of life.

Keywords Myelomeningocele $\cdot$ Neurosurgery

Hydrocephalus · Arnold-Chiari malformation · Spina bifida

\section{Abbreviations}

(MMC) Myelomeningocele

(CSF) Cerebrospinal fluid

(CNS) Central nervous system

\section{Introduction}

Neural tube closure defects are congenital malformations of the central nervous system (CNS) that occur in $0.5-8$ of 1000 live births [1]. Myelomeningocele (MMC) is the most common type of these defects. In the United States of America, the incidence of MMC has remained stable at 0.34 cases per 1000 live births after the introduction of folic acid fortification [2]. Case series in Brazil have reported prevalence rates ranging from 0.6 to 13.1 cases per 1000 live births among different regions [3, 4]. In most cases, MMC is an isolated malformation with a multifactorial origin [5], but it is sometimes 


\section{Author's personal copy}

associated with genetic syndromes, chromosomal abnormalities, or environmental exposure [6].

MMC may present as flat dysplastic tissue or as a cystic lesion with a herniary sac protruding through the vertebral bone defect containing meninges, spinal cord, and cerebrospinal fluid (CSF). The neurological impairments associated with MMC are primarily caused by defective spinal cord and nerve root development, and secondarily caused by injury to these structures following exposure to amniotic fluid, direct trauma, or infection [7].

Spinal cord damage is not the only cause of the neurological impairments in MMC. Other anomalies, including hydrocephalus and Arnold-Chiari malformation, also contribute to the clinical features of MMC [8]. In the neonatal period, brainstem dysfunction is manifested by laryngeal stridor, central apnea, and disturbed feeding. Brainstem dysfunction, CNS infection, and neurosurgical complications such as ventricular shunt malfunction and wound infection are the main contributors to the unfavorable outcomes of MMC [9].

Advances in the treatment of MMC and associated developmental brain abnormalities, particularly early surgical intervention and implantation of ventricular shunts, have improved the survival outcomes of MMC [10,11]. Primary failure of neural tube closure exposes the spinal cord to external environmental factors. The early repair of MMC avoids secondary damage to a relatively normal spinal cord that is without protective tissue coverage. Early ventricular shunt placement facilitates functional recovery, reduces the ventricular size, and reconstitutes the cortical mantle [12]. Clinical improvements in neurological function occur as a result of the functional improvements of the remaining neurons. Nonetheless, the response to surgical treatment is usually poor in cases with established neurological damage caused by chronic brain stem compression, ischemia, or brain stem dysgenesis [13].

MMC is associated with high rates of death and neurological disabilities, which are correlated with the anatomical characteristics of the defect and the development of acquired complications [14]. The current challenge for postnatal management of $\mathrm{MMC}$ is the early recognition of cases at high risk of developing complications, in order to promptly establish individualized treatment strategies.

The aim of this study was to identify short-term prognostic markers relevant to neonates with MMC. To achieve this aim, we analyzed the anatomical characteristics of the spinal defect and the technical aspects of the neurosurgical correction in neonates with MMC.

\section{Methods}

The research ethics committee of our institution approved this study. We conducted a retrospective cohort study of neonates with thoracic, lumbar, or sacral MMC who were born between
January 2007 and December 2013 at the Neonatal Center of the Children's Institute, University of São Paulo. Patients with congenital infection, chromosomal abnormalities, and other major malformations unrelated to MMC were excluded from this study.

We evaluated the following features of the anatomy and neurosurgical treatment of MMC: spinal level and size of the defect, antenatal hydrocephalus, macrocrania at birth, rupture of MMC membranes, and the moment of surgical correction. The following prognostic markers were also retrieved: the need for neonatal resuscitation, the length of hospital stay, the need for a ventricular shunt, wound dehiscence, wound infection, CNS infection, and sepsis. Macrocrania was defined as a head circumference $>97$ th percentile for gestational age at birth according to the Fenton growth chart [15]. Rupture of the MMC sac was defined as visual observation of spontaneous CSF leakage.

We adopted a qualitative classification of the MMC lesions based on the surgical technique used to repair the lesions. Lesions were classified as large if the repair required soft tissue closure with muscle and fascial flap techniques to provide a tension-free defect cover. Lesions were classified as small if they were corrected by primary closure. Prompt repair of the MMC was defined as an intervention performed immediately after birth, before admission to the intensive care unit. All of the neonates received prophylactic antibiotics, which were started preoperatively. A ventricular shunt was placed if CSF persistently leaked from the MMC wound, if the fontanelle was bulged and firm, and if progressive ventricular enlargement was observed on serial ultrasound studies.

Wound infection was defined as any of the following: (a) purulent drainage from the incision; (b) if the surgical wound was re-opened by the surgeon in the case of fever, localized sensitivity, and pain; (c) diagnosis of an abscess intraoperatively or histopathologically; or (d) if the surgeon suspected the presence of infection [16]. Ventriculitis was defined as a positive culture or positive bacterioscopic examination of the CSF, and/or the development of infection-related symptoms associated with changes in CSF biochemistry [16].

Quantitative variables are presented using summary measures (mean, standard deviation, median, range) and qualitative variables as absolute and relative frequencies. Relationships between qualitative outcomes and factors of interest were examined using $\chi^{2}$ or Fisher's exact tests. The relationships with the quantitative outcome duration of hospital stay were evaluated using the Mann-Whitney test [17]. Relative risks were estimated with $95 \%$ confidence intervals [18]. Multivariate linear regression was used to evaluate the quantitative outcomes and a Poisson regression model was used for the qualitative outcomes [19]. 


\section{Author's personal copy}

\section{Results}

Between January 2007 and December 2013, 12,559 neonates were born at our institution. Eighty neonates were diagnosed with MMC, resulting in a incidence of 6.4 cases per 1000 live births. Ten neonates with MMC were excluded from the present analyses owing to cervical MMC $(n=1)$, congenital heart disease $(n=4)$, trisomy $13(n=1)$, omphalocele $(n=3)$, and encephalocele $(n=1)$.

The baseline characteristics of the pregnant women and the neonates are presented in Tables 1 and 2, respectively. The anatomical and surgical characteristics of MMC are presented in Table 3.

The early complications of MMC in the 70 neonates included wound infection in $9(13 \%)$, wound dehiscence in 27 (39\%), bacterial ventriculitis in $11(16 \%)$, and sepsis in 20 $(29 \%)$. The mean hospital stay was 28 days (range, 4196 days). Shunted hydrocephalus occurred in 32 neonates $(46 \%)$. Eight $(25 \%)$ neonates underwent ventricular shunt revision. Three $(4.28 \%$ ) neonates with MMC died during the study period. One neonate died 4 days after delivery owing to aspiration pneumonia. The other two deaths were related to sepsis and CNS infection (Table 4).

Of the 11 neonates diagnosed with ventriculitis, bacteria were isolated from the CSF in three cases, with Acinetobacter baumannii, Klebisiella pneumonia, and Enterobacter cloacae in one patient each. Neonates with ventriculitis were given empirical antibiotics comprising intravenous vancomycin and

\section{Table 1 Baseline maternal characteristics}

\begin{tabular}{ll}
\hline Characteristic & Frequency number (\%) \\
Previous pregnancies & $25(36)$ \\
0 & $22(31)$ \\
1 & $23(33)$ \\
2 or more & $29(41)$ \\
Nullipara & \\
Mode of delivery & $0(0)$ \\
Vaginal & $70(100)$ \\
Cesarean section & \\
Maternal comorbidities & $2(3)$ \\
Epilepsy & $2(3)$ \\
Asthma & $3(4)$ \\
Hypothyroidism & $2(3)$ \\
Diabetes mellitus & $0(0)$ \\
Spontaneous membrane rupture & $0(0)$ \\
Premature labor & $3(4)$ \\
Acute fetal distress & $7(10)$ \\
Preeclampsia or gestational hypertension & Average/median (range) \\
Characteristic & $27 / 26(15-43)$ \\
Maternal age (years) & $38 / 38(31-41)$ \\
Gestational age (weeks) & \\
\hline &
\end{tabular}

\begin{tabular}{ll} 
Table $2 \quad$ Baseline newborn characteristics & \\
\hline Characteristics & $\begin{array}{l}\text { Frequency number }(\%) \\
\text { Female }\end{array}$ \\
Gestational age vs. birth weight & $41(59)$ \\
$\quad$ Small for gestational age & $8(11)$ \\
$\quad$ Appropriate for gestational age & $61(87)$ \\
$\quad$ Large for gestational age & $1(1)$ \\
Gestational age $<37$ weeks & $6(9)$ \\
Characteristics & Average/median (range) \\
Head circumference $(\mathrm{cm})$ & $35 / 35(28-50)$ \\
Birth weight $(\mathrm{g})$ & $3024 / 3065(880-4150)$
\end{tabular}

meropenem or cefepime before the results of the culture tests. The mean duration of intravenous antibiotics was 23.6 days. External ventricular drainage was performed in seven neonates. The mean hospital stay was 2.5 times longer in neonates diagnosed with ventriculitis compared with the mean for the overall study group (69.3 versus 28 days, respectively).

The results of the multivariate analysis are presented in Table 5. Large MMC was associated with CNS infection, wound complications, and longer hospital stay. The mean hospital stay was 39 days longer in patients with thoracic $\mathrm{MMC}$ than that in the patients with lumbar or sacral MMC.

All neonates with MMC received prophylactic antibiotics. $\mathrm{MMC}$ surgical repair performed $48 \mathrm{~h}$ after birth increased the risk of CNS infection by 5.72 times compared with surgical repair performed before $48 \mathrm{~h}$. Prompt surgical repair showed no additional benefit in terms of short-term outcomes than surgical repair within the first $48 \mathrm{~h}$.

Table 3 Anatomical characteristics of the myelomeningocele and its neurosurgical correction

\begin{tabular}{ll}
\hline Characteristics & Frequency number (\%) \\
Spinal level & \\
Thoracic & $4(6)$ \\
Lumbar & $59(84)$ \\
Sacral & $5(7)$ \\
$\quad$ Not classified & $2(3)$ \\
Large defects & $11(16)$ \\
Antenatal hydrocephalus & $54(76)$ \\
Macrocrania & $11(16)$ \\
Rupture of MMC membranes & $45(64)$ \\
Surgical intervention & \\
$0-24$ h & $46(66)$ \\
$25-48$ h & $14(20)$ \\
$49-72$ h & $4(6)$ \\
$>72$ h & $6(9)$ \\
Prompt repair surgery & $27(39)$ \\
Characteristics & Average/median (range) \\
Surgical intervention (h) & $26 / 8(1-201)$ \\
\hline
\end{tabular}




\section{Author's personal copy}

Childs Nerv Syst

\begin{tabular}{ll} 
Table 4 & Description of short-term outcomes \\
\hline Outcomes & Frequency number (\%) \\
Ventricular shunt & $32(46)$ \\
Ventricular shunt revision & $8(25)$ \\
External ventricular drainage & $9(13)$ \\
Wound infection & $9(13)$ \\
Wound dehiscence & $27(39)$ \\
Sepsis & $20(29)$ \\
Central nervous system infection & $11(16)$ \\
Death & $3(4)$ \\
Outcomes & Average/median (range) \\
Length hospital stay (days) & $28 / 21(4-196)$ \\
\hline
\end{tabular}

The absence of antenatal hydrocephalus was a favorable prognostic marker. The prevalence of a combination of the need for ventricular shunt, surgical wound complications, CNS infection, and the need for neonatal resuscitation was $70 \%$ lower in neonates without antenatal hydrocephalus than that in the neonates with this disorder.

\section{Discussion}

The anatomical characteristics of the spinal defect and its neurosurgical correction were evaluated in order to identify potential short-term prognostic markers of MMC. In particular, large spinal defects were associated with unfavorable outcomes because neonates with a large MMC were 5.14 times more likely to develop CNS infection and 7.98 times more likely to develop wound infection than patients with a small MMC. In addition, the mean hospital stay was 32.16 days longer in patients with a large MMC.

CNS infection and wound infection occurred in 15.71 and $12.8 \%$ of all neonates, respectively. In a retrospective study by Demir et al., the rates of CNS infection and wound infection were higher (19.8 and $17.6 \%$, respectively) than those in our study [20]. In accordance with our findings, neonates with larger spinal defects had a greater risk of wound infection; however, Demir et al. did not describe this risk factor as a marker for CNS infection.

The location of the spinal defect is clinically relevant. Lesions involving higher levels of the spinal cord and nerve roots are associated with worse neurological prognosis than lesions at lower levels. In the long term, the level of the spinal cord injury may affect academic, neurobehavioral, and intellectual performances. This association was shown in a study published in 2005 by Fletcher et al., who evaluated 268 children with MMC at 7-16 years of age [21].

Furthermore, Rintoul et al. conducted a retrospective analysis of 297 patients with spina bifida and reported that a ventricular shunt was required more frequently in patients with
Table 5 Relationships between prognostic factors and short-term outcomes

\begin{tabular}{|c|c|c|}
\hline & $p$ & IRR (IC $95 \%$ ) \\
\hline \multicolumn{3}{|l|}{ Neonatal resuscitation } \\
\hline Spinal level: sacral/lumbar & 0.34 & $2.83(0.34-23.73)$ \\
\hline Size of the defect: large defects & 0.08 & $2.79(0.87-8.94)$ \\
\hline Gestational age & 0.40 & $0.88(0.66-1.18)$ \\
\hline Macrocrania: present & 0.14 & $2.46(0.75-8.09)$ \\
\hline Acute fetal distress: present & 0.65 & $1.72(0.16-18.05)$ \\
\hline \multicolumn{3}{|l|}{ Ventricular shunt } \\
\hline Spinal level: sacral/lumbar & 0.90 & $1.10(0.24-4.94)$ \\
\hline Size of the defect: large defects & 0.39 & $1.48(0.60-3.61)$ \\
\hline Surgical repair: $>48 \mathrm{~h}$ & 0.72 & $0.80(0.24-2.67)$ \\
\hline Antenatal hydrocephalus: present & 0.08 & $2.90(0.87-9.65)$ \\
\hline \multicolumn{3}{|l|}{ Wound infection } \\
\hline Spinal level: sacral/lumbar & 0.90 & $1.15(0.13-9.80)$ \\
\hline Size of the defect: large defects & 0.004 & $7.98(1.93-33.02)$ \\
\hline Surgical repair: $>48 \mathrm{~h}$ & 0.83 & $0.77(0.08-7.77)$ \\
\hline Antenatal hydrocephalus: present & 0.69 & $0.68(0.11-4.25)$ \\
\hline \multicolumn{3}{|l|}{ Wound dehiscence } \\
\hline Spinal level: sacral/lumbar & 0.86 & $1.14(0.25-5.08)$ \\
\hline Size of the defect: large defects & 0.04 & $2.51(1.05-6.02)$ \\
\hline Surgical repair: $>48 \mathrm{~h}$ & 0.54 & $1.39(0.47-4.14)$ \\
\hline Antenatal hydrocephalus: present & 0.35 & $1.67(0.56-4.96)$ \\
\hline \multicolumn{3}{|l|}{ Central nervous system infection } \\
\hline Spinal level: sacral/lumbar & 0.55 & $0.59(0.11-3.24)$ \\
\hline Size of the defect: large defects & 0.01 & $5.14(1.37-19.35)$ \\
\hline Surgical repair: $>48 \mathrm{~h}$ & 0.03 & $5.72(1.14-28.58)$ \\
\hline Rupture MMC sac: present & 0.11 & $4.17(0.73-23.87)$ \\
\hline Gestational age & 0.93 & $1.01(0.75-1.37)$ \\
\hline \multicolumn{3}{|l|}{ Neonatal sepsis } \\
\hline Spinal level: sacral/lumbar & 0.31 & $0.49(0.12-1.97)$ \\
\hline Size of the defect: large defects & 0.14 & $2.20(0.77-6.32)$ \\
\hline Surgical repair: $>48 \mathrm{~h}$ & 0.92 & $0.92(0.18-4.64)$ \\
\hline Rupture MMC sac: present & 0.59 & $1.35(0.45-4.03)$ \\
\hline Gestational age & 0.97 & $1.01(0.74-1.37)$ \\
\hline \multicolumn{3}{|l|}{ Favorable prognostic marker } \\
\hline Spinal level: sacral/lumbar & 0.79 & $1.37(0.13-14.38)$ \\
\hline Size of the defect: large defects & 0.24 & $0.30(0.04-2.27)$ \\
\hline Surgical repair: $>48 \mathrm{~h}$ & 0.25 & $1.92(0.63-5.84)$ \\
\hline Rupture MMC sac: present & 0.84 & $1.09(0.45-2.65)$ \\
\hline Gestational age & 0.77 & $1.04(0.80-1.35)$ \\
\hline \multirow[t]{2}{*}{ Antenatal hydrocephalus: present } & 0.01 & $0.35(0.15-0.81)$ \\
\hline & $p$ & $\beta($ IC $95 \%)$ \\
\hline \multicolumn{3}{|l|}{ Length hospital stay } \\
\hline Spinal level: sacral/lumbar & 0.004 & $\begin{array}{c}-39.89[-66.17- \\
(-13.61)]\end{array}$ \\
\hline Size of the defect: large defects & $<0.001$ & $32.16(15.28-49.04)$ \\
\hline Surgical repair: $>48 \mathrm{~h}$ & 0.76 & $-2.68(-20.54-15.18)$ \\
\hline Gestational age & 0.43 & $-1.52(-5.38-2.33)$ \\
\hline
\end{tabular}




\section{Author's personal copy}

thoracic injury. The procedure was performed in $97 \%$ of their patients with thoracic MMC. Notably, a ventricular shunt was inserted into 87 and $37 \%$ of patients with lumbar or sacral MMC, respectively. The procedure was conducted in the first week of life in most cases, at a median age of 5 days [22]. In our series, neonates with thoracic lesions showed no additional need for a ventricular shunt or increased risk of infectious complications. However, the mean hospital stay was 2.5 times longer in neonates with thoracic lesions than that in neonates with lumbar or sacral lesions. Although the need for a ventricular shunt was not increased in neonates with thoracic lesions, this outcome was only assessed up to discharge from our institution. The median follow-up time of these neonates was 21 days, which is greater than the median age at which a ventricular shunt was inserted in the study by Rintoul et al. [22]. Considering these results, we believe there was a reduced need for a ventricular shunt among the neonates in our study.

Most patients with MMC are diagnosed with hydrocephalus in the antenatal period. However, only one in eight newborns has a head circumference $>98$ th percentile at birth, and only $16 \%$ of them manifest clinical intracranial hypertension [23]. Fifty-four $(75.71 \%)$ of the neonates in our study were diagnosed with hydrocephalus in the antenatal period, and $11(20.37 \%)$ were diagnosed with macrocrania at birth. Neonates without antenatal hydrocephalus were 1.73 times less likely to develop the combination of surgical wound complications, CNS infection, need for a ventricular shunt, and resuscitation in the delivery room.

The risk of CNS infection was 5.72 times lower in patients who underwent surgical correction of the defect $<48 \mathrm{~h}$ after delivery. This finding is consistent with the results of a prospective study by Bulbul et al. in 2010 that described higher rates of CNS infection and antibiotic therapy, and longer hospital stay if surgical interventions are performed $>3$ days after delivery [10].

The theoretical advantages of very early surgical correction (i.e., prompt repair) include reduced spinal cord exposure, lower incidence of hydroelectrolyte disorders caused by CSF loss, and reduced risk of MMC sac rupture. In a case-control study published by Pinto et al. in 2009, immediate surgical repair of MMC was compared to a historical control of postnatal surgical correction performed after admission to an intensive care unit. The authors reported that prompt MMC repair was associated with a lower incidence of surgical wound dehiscence and better neurodevelopmental outcomes in the first year of life [24]. We found no additional benefits of prompt repair when compared to early surgical intervention (i.e., within the first $48 \mathrm{~h}$ ) in terms of CNS infection or any of the other outcomes analyzed in this cohort of neonates.

Intrauterine correction has been performed in some centers in the last 15 years [25]. This approach minimizes the incidence of complications caused by lower secondary spinal cord injury, reduces the incidence of Arnold-Chiari malformation, and reduces the need for a ventricular shunt to treat hydrocephalus [26]. However, the high costs of antenatal procedures, maternal risks, and the possibility of complications affecting current or future pregnancies limit the widespread use of this technique. The American College of Obstetricians and Gynecologists recommends that fetal surgery should only be offered at highly experienced centers, with trained multidisciplinary staff and facilities to provide adequate postoperative intensive care support [27].

\section{Limitations}

Classification of the MMC defect was based on medical records. Because of the retrospective design of this study, accurate measurements of the defect size were not available. Therefore, we classified defect size qualitatively according to the surgical technique required for MMC closure. Furthermore, the medical records did not include differentiation of spinal level based on bony vertebral defect or functional neurological assessments. A more precise classification of lesions with respect to neurological spinal cord impairment could be useful for determining the short-term prognosis, as it is already known from previous studies [22].

\section{Conclusion}

The extent of the spinal cord defect and the timing of surgical correction influenced the short-term outcomes of neonates with MMC. Large lesions were associated with higher rates of CNS infection and surgical wound complications, and longer hospital stay. Interventions performed $<48 \mathrm{~h}$ after delivery significantly reduced the frequency of CNS infection. The absence of antenatal hydrocephalus was associated with fewer complications in the first days of life.

Compliance with ethical standards The research ethics committee of our institution approved this study.

Financial disclosure The authors have no financial relationships relevant to this article to disclose.

Funding source This research had no financial support.

Conflict of interest The authors declare that they have no competing interests.

\section{References}

1. Saitsu H, Yamada S, Uwabe C, Ishibashi M, Shiota K (2004) Development of the posterior neural tube in human embryos. Anat Embryol (Berl) 209(2):107-117 
2. Boulet SL, Yang Q, Mai C, Kirby RS, Collins JS, Robbins JM et al (2008) Trends in the postfortification prevalence of spina bifida and anencephaly in the United States. Birth Defects Res A Clin Mol Teratol 82(7):527-532

3. Nascimento LFC (2008) Prevalência de defeitos de fechamento de tubo neural no Vale do Paraíba. São Paulo Revista Paulista de Pediatria 26:372-377

4. Cunha CJ, Fontana T, Garcias GL, Martino-Roth MDG (2005) Fatores genéticos e ambientais associados a espinha bífida. Revista Brasileira de Ginecologia e Obstetrícia 27:268-274

5. Copp AJ, Stanier P, Greene ND (2013) Neural tube defects: recent advances, unsolved questions, and controversies. Lancet Neurol 12(8):799-810

6. Moretti ME, Bar-Oz B, Fried S, Koren G (2005) Maternal hyperthermia and the risk for neural tube defects in offspring: systematic review and meta-analysis. Epidemiology 16(2):216-219

7. Meuli M, Moehrlen U (2014) Fetal surgery for myelomeningocele is effective: a critical look at the whys. Pediatr Surg Int 30(7):689697

8. Adzick NS, Walsh DS (2003) Myelomeningocele: prenatal diagnosis, pathophysiology and management. Semin Pediatr Surg 12(3): $168-174$

9. Hahn YS (1995) Open myelomeningocele. Neurosurg Clin N Am 6(2):231-241

10. Bulbul A, Can E, Bulbul LG, Cömert S, Nuhoglu A (2010) Clinical characteristics of neonatal meningomyelocele cases and effect of operation time on mortality and morbidity. Pediatr Neurosurg 46(3):199-204

11. Piatt JH Jr (2010) Treatment of myelomeningocele: a review of outcomes and continuing neurosurgical considerations among adults. J Neurosurg Pediatr 6(6):515-525

12. Venkataramana NK, Mukundan CR (2011) Evaluation of functional outcomes in congenital hydrocephalus. J Pediatr Neurosci 6(1): 4-12

13. Pollack IF, Kinnunen D, Albright AL (1996) The effect of early craniocervical decompression on functional outcome in neonates and young infants with myelodysplasia and symptomatic Chiari II malformations: results from a prospective series. Neurosurgery 38(4):703-710, discussion 10

14. Oakeshott P, Hunt GM, Poulton A, Reid F (2010) Expectation of life and unexpected death in open spina bifida: a 40-year complete, non-selective, longitudinal cohort study. Dev Med Child Neurol 52(8):749-753
15. Fenton TR (2003) A new growth chart for preterm babies: Babson and Benda's chart updated with recent data and a new format. BMC Pediatr 3:13

16. Horan TC, Andrus M, Dudeck MA (2008) CDC/NHSN surveillance definition of health care-associated infection and criteria for specific types of infections in the acute care setting. Am J Infect Control 36(5):309-332

17. Kirkwood BR, Sterne JAC, ebrary Inc. Essential medical statistics. Malden, Mass.: Blackwell Pub.,; 2003. Available from: http://site. ebrary.com/lib/yale/Doc?id=10419207

18. Hosmer DW, Lemeshow S (2000) Applied logistic regression. 2nd ed. New York: Wiley. xii, $375 \mathrm{pp}$

19. McCullagh P, Nelder JA (1989) Generalized linear models. 2nd ed London; New York: Chapman \& Hall/CRC. xix, 511 pp

20. Demir N, Peker E, Gülşen İ, Ağengin K, Tuncer O (2015) Factors affecting infection development after meningomyelocele repair in newborns and the efficacy of antibiotic prophylaxis. Childs Nery Syst

21. Fletcher JM, Copeland K, Frederick JA, Blaser SE, Kramer LA, Northrup H et al (2005) Spinal lesion level in spina bifida: a source of neural and cognitive heterogeneity. J Neurosurg 102(3 Suppl): $268-279$

22. Rintoul NE, Sutton LN, Hubbard AM, Cohen B, Melchionni J, Pasquariello PS et al (2002) A new look at myelomeningoceles: functional level, vertebral level, shunting, and the implications for fetal intervention. Pediatrics 109(3):409-413

23. Tamburrini G, Frassanito P, Iakovaki K, Pignotti F, Rendeli C, Murolo D et al (2013) Myelomeningocele: the management of the associated hydrocephalus. Childs Nerv Syst 29(9):1569-1579

24. Pinto FC, Matushita H, Furlan AL, Alho EJ, Goldenberg DC, Bunduki V et al (2009) Surgical treatment of myelomeningocele carried out at 'time zero' immediately after birth. Pediatr Neurosurg 45(2):114-118

25. Adzick NS, Sutton LN, Crombleholme TM, Flake AW (1998) Successful fetal surgery for spina bifida. Lancet 352(9141):16751676

26. Adzick NS, Thom EA, Spong CY, Brock JW, Burrows PK, Johnson MP et al (2011) A randomized trial of prenatal versus postnatal repair of myelomeningocele. N Engl J Med 364(11):993-1004

27. American College of O, Gynecologists (2013) ACOG Committee opinion no. 550: maternal-fetal surgery for myelomeningocele. Obstet Gynecol 121(1):218-219 


\section{REFERÊNCIAS}


1. Robbins SL, Kumar V. Robbins and Cotran pathologic basis of disease. 8th ed. Philadelphia, Saunders/Elsevier; 2010.

2. Boerma JT, Fat DM, Mathers C, ebrary Inc., World Health Organization. The global burden of disease 2004 update. Geneva: World Health Organization; 2008. [Cited 2015 jan 28] Available from: http://www.who.int/healthinfo/global_burden_disease/GBD_report_2004upd ate_full.pdf

3. Saitsu H, Yamada S, Uwabe C, Ishibashi M, Shiota K. Development of the posterior neural tube in human embryos. Anat Embryol (Berl). 2004;209(2):107-17.

4. Volpe JJ. Neurology of the newborn. 5th eddition. Philadelphia: Saunders/Elsevier; 2008. Chap.1; Neural Tube Formation and Prosencephalic Development. p.3-50.

5. Moretti ME, Bar-Oz B, Fried S, Koren G. Maternal hyperthermia and the risk for neural tube defects in offspring: systematic review and meta-analysis. Epidemiology. 2005;16(2):216-9.

6. Copp AJ, Stanier P, Greene ND. Neural tube defects: recent advances, unsolved questions, and controversies. Lancet Neurol. 2013;12(8):799-810.

7. Dolk H, Loane M, Garne E. The prevalence of congenital anomalies in Europe. Adv Exp Med Biol. 2010;686:349-64.

8. Lammer EJ, Sever LE, Oakley GP. Teratogen update: valproic acid. Teratology. 1987;35(3):465-73. 
9. Soler NG, Walsh $\mathrm{CH}$, Malins JM. Congenital malformations in infants of diabetic mothers. Q J Med. 1976;45(178):303-13.

10. Rasmussen SA, Chu SY, Kim SY, Schmid CH, Lau J. Maternal obesity and risk of neural tube defects: a metaanalysis. Am J Obstet Gynecol. 2008;198(6):611-9.

11. Detrait ER, George TM, Etchevers HC, Gilbert JR, Vekemans M, Speer MC. Human neural tube defects: developmental biology, epidemiology, and genetics. Neurotoxicol Teratol. 2005;27(3):515-24.

12. Imbard A, Benoist JF, Blom HJ. Neural tube defects, folic acid and methylation. Int J Environ Res Public Health. 2013;10(9):4352-89.

13. Oakley GP. The scientific basis for eliminating folic acid-preventable spina bifida: a modern miracle from epidemiology. Ann Epidemiol. 2009;19(4):226-30.

14. Greene ND, Stanier P, Moore GE. The emerging role of epigenetic mechanisms in the etiology of neural tube defects. Epigenetics. $2011 ; 6(7): 875-83$.

15. Lumley J, Watson L, Watson M, Bower C. Periconceptional supplementation with folate and/or multivitamins for preventing neural tube defects. Cochrane Database Syst Rev. 2001(3):CD001056.

16. Davis RL, Robertson DM. Textbook of neuropathology. Baltimore: Williams \& Wilkins; 1985.

17. Mitchell LE, Adzick NS, Melchionne J, Pasquariello PS, Sutton LN, Whitehead AS. Spina bifida. Lancet. 2004;364(9448):1885-95.

18. Bowman RM, Boshnjaku V, McLone DG. The changing incidence of myelomeningocele and its impact on pediatric neurosurgery: a review from the Children's Memorial Hospital. Childs Nerv Syst. 2009;25(7):801-6. 
19. Boulet SL, Yang Q, Mai C, Kirby RS, Collins JS, Robbins JM, Meyer R, Canfield MA, Mulinare J; National Birth Defects Prevention Network. Trends in the postfortification prevalence of spina bifida and anencephaly in the United States. Birth Defects Res A Clin Mol Teratol. 2008;82(7):527-32.

20. Nascimento LFC. Prevalência de defeitos de fechamento de tubo neural no Vale do Paraíba, São Paulo. Rev Paul Pediatr. 2008;26(4):372-7.

21. Cunha CJ, Fontana T, Garcias GdL, Martino-Roth MdG. Fatores genéticos e ambientais associados a espinha bífida. Rev Bras Ginecol Obstet. $2005 ; 27(5): 268-74$.

22. Pinto FCG KV, Tanaka T, Matoshita H, Calil VMLT, Vaz FAC. Caracterização das malformações congênitas neurocirurgicas em Centro de Referência Terciário. Anais do XIX Congresso Brasileiro de Perinatologia,XVI Reunião de Enfermagem Perinatal, I Reunião multidisciplinar Perinatal; Fortaleza, 2007.

23. Bronsteen RA, Comstock $\mathrm{CH}$. Central nervous system anomalies. Clin Perinatol. 2000;27(4):791-812.

24. Meuli M, Moehrlen U. Fetal surgery for myelomeningocele is effective: a critical look at the whys. Pediatr Surg Int. 2014;30(7):689-97.

25. Hutchins GM, Meuli M, Meuli-Simmen C, Jordan MA, Heffez DS, Blakemore KJ. Acquired spinal cord injury in human fetuses with myelomeningocele. Pediatr Pathol Lab Med. 1996;16(5):701-12.

26. Adzick NS, Walsh DS. Myelomeningocele: prenatal diagnosis, pathophysiology and management. Semin Pediatr Surg. 2003;12(3):168-74.

27. Rekate HL. A contemporary definition and classification of hydrocephalus. Semin Pediatr Neurol. 2009;16(1):9-15. 
28. Arslan M, Eseoglu M, Gudu BO, Demir I, Kozan A, Gokalp A, Sosuncu E, Kiymaz N. Comparison of simultaneous shunting to delayed shunting in infants with myelomeningocele in terms of shunt infection rate. Turk Neurosurg. 2011;21(3):397-402.

29. Dias MS, McLone DG. Hydrocephalus in the child with dysraphism. Neurosurg Clin North Am. 1993;4(4):715-26.

30. Mandell JG, Kulkarni AV, Warf BC, Schiff SJ. Volumetric brain analysis in neurosurgery: Part 2. Brain and CSF volumes discriminate neurocognitive outcomes in hydrocephalus. J Neurosurg Pediatr. 2015;15(2):125-32.

31. Rintoul NE, Sutton LN, Hubbard AM, Cohen B, Melchionni J, Pasquariello PS, Adzick NS. A new look at myelomeningoceles: functional level, vertebral level, shunting, and the implications for fetal intervention. Pediatrics. 2002;109(3):409-13.

32. Prigatano GP, Zeiner HK, Pollay M, Kaplan RJ. Neuropsychological functioning in children with shunted uncomplicated hydrocephalus. Childs Brain. 1983;10(2):112-20.

33. Venkataramana NK, Mukundan CR. Evaluation of functional outcomes in congenital hydrocephalus. J Pediatr Neurosci. 2011;6(1):4-12.

34. Stevenson KL. Chiari Type II malformation: past, present, and future. Neurosurg Focus. 2004;16(2):E5.

35. Dahl M, Ahlsten G, Carlson H, Ronne-Engstrom E, Lagerkvist B, Magnusson G, Norrlin S, Olsen L, Stromberg B, Thuomas KA. Neurological dysfunction above cele level in children with spina bifida cystica: a prospective study to three years. Dev Med Child Neurol. 1995;37(1):30-40.

36. Hahn YS. Open myelomeningocele. Neurosurg Clin North Am. 1995;6(2):231-41. 
37. Dias MS. Neurosurgical management of myelomeningocele (spina bifida). Pediatr Rev. 2005;26(2):50-60; discussion 50-60.

38. Nagler J, Levy JA, Bachur RG. Stridor in an infant with myelomeningocele. Pediatr Emerg Care. 2007;23(7):478-81.

39. Tomita T, McLone DG. Acute respiratory arrest. A complication of malformation of the shunt in children with myelomeningocele and ArnoldChiari malformation. Am J Dis Child. 1983;137(2):142-4.

40. McLone DG, Dias MS. The Chiari II malformation: cause and impact. Childs Nerv Syst. 2003;19(7-8):540-50.

41. Pollack IF, Pang D, Albright AL, Krieger D. Outcome following hindbrain decompression of symptomatic Chiari malformations in children previously treated with myelomeningocele closure and shunts. $J$ Neurosurg. 1992;77(6):881-8.

42. Park TS, Hoffman HJ, Hendrick EB, Humphreys RP. Experience with surgical decompression of the Arnold-Chiari malformation in young infants with myelomeningocele. Neurosurgery. 1983;13(2):147-52.

43. Bulbul A, Can E, Bulbul LG, Comert S, Nuhoglu A. Clinical characteristics of neonatal meningomyelocele cases and effect of operation time on mortality and morbidity. Pediatr Neurosurg. 2010;46(3):199-204.

44. Piatt JH, Jr. Treatment of myelomeningocele: a review of outcomes and continuing neurosurgical considerations among adults. J Neurosurg Pediatr. 2010;6(6):515-25.

45. Pinto FC, Matushita H, Furlan AL, Alho EJ, Goldenberg DC, Bunduki V, Krebs VL, Teixeira MJ. Surgical treatment of myelomeningocele carried out at 'time zero' immediately after birth. Pediatr Neurosurg. 2009;45(2):114-8.

46. Adzick NS, Sutton LN, Crombleholme TM, Flake AW. Successful fetal surgery for spina bifida. Lancet. 1998;352(9141):1675-6. 
47. Adzick NS, Thom EA, Spong CY, Brock JW, Burrows PK, Johnson MP, Howell LJ, Farrell JA, Dabrowiak ME, Sutton LN, Gupta N, Tulipan NB, DÁlton ME, Farmer DL: MOMS Investigators. A randomized trial of prenatal versus postnatal repair of myelomeningocele. $N$ Engl $J$ Med. 2011;364(11):993-1004.

48. American College of O, Gynecologists. ACOG Committee opinion no. 550: maternal-fetal surgery for myelomeningocele. Obstet Gynecol. 2013;121(1):218-9.

49. Fenton TR. A new growth chart for preterm babies: Babson and Benda's chart updated with recent data and a new format. BMC Pediatr. 2003;3:13.

50. Horan TC, Andrus M, Dudeck MA. CDC/NHSN surveillance definition of health care-associated infection and criteria for specific types of infections in the acute care setting. Am J Infect Control. 2008;36(5):309-32.

51. Goldstein B, Giroir B, Randolph A, International Consensus Conference on Pediatric S. International pediatric sepsis consensus conference: definitions for sepsis and organ dysfunction in pediatrics. Pediatr Crit Care Med. 2005;6(1):2-8.

52. Kirkwood BR, Sterne JAC, ebrary Inc. Essential medical statistics. Malden: Blackwell Pub.; 2003.

53. Hosmer DW, Lemeshow S. Applied logistic regression. 2nd ed. New York: Wiley; 2000.

54. McCullagh P, Nelder JA. Generalized linear models. 2nd ed. London ; New York: Chapman \& Hall/CRC; 1989.

55. de Araújo AA, de Souza GL, Brandão GHA, Oliveira YMdC, do Nascimento HG, Alves MdSCF. Prevalência e caracterização dos casos de mielomeningocele no Rio Grande do Norte. Rev Ciênc Med. 2012;21(16):55-61. 
56. Whiteman D, Murphy M, Hey K, O'Donnell M, Goldacre M. Reproductive factors, subfertility, and risk of neural tube defects: a case-control study based on the Oxford Record Linkage Study Register. Am J Epidemiol. 2000;152(9):823-8.

57. Vieira AR, Castillo Taucher S. [Maternal age and neural tube defects: evidence for a greater effect in spina bifida than in anencephaly]. Rev Med Chil. 2005;133(1):62-70.

58. Frey L, Hauser WA. Epidemiology of neural tube defects. Epilepsia. 2003;44(Suppl 3):4-13.

59. De Marco P, Merello E, Calevo MG, Mascelli S, Pastorino D, Crocetti L, De Biasio P, Piatelli G, Cama A, Capra V. Maternal periconceptional factors affect the risk of spina bifida-affected pregnancies: an Italian case-control study. Childs Nerv Syst. 2011;27(7):1073-81.

60. Hendricks KA, Simpson JS, Larsen RD. Neural tube defects along the Texas-Mexico border, 1993-1995. Am J Epidemiol. 1999;149(12):1119-27.

61. Shaw GM, Velie EM, Schaffer D. Risk of neural tube defect-affected pregnancies among obese women. JAMA. 1996;275(14):1093-6.

62. Hendricks KA, Nuno OM, Suarez L, Larsen R. Effects of hyperinsulinemia and obesity on risk of neural tube defects among Mexican Americans. Epidemiology. 2001;12(6):630-5.

63. Kiely M. Reproductive and perinatal epidemiology. Boca Raton: CRC Press; 1991.

64. Petrova JG, Vaktskjold A. The incidence of neural tube defects in Norway and the Arkhangelskaja Oblast in Russia and the association with maternal age. Acta Obstet Gynecol Scand. 2009;88(6):667-72. 
65. Williams LJ, Rasmussen SA, Flores A, Kirby RS, Edmonds LD. Decline in the prevalence of spina bifida and anencephaly by race/ethnicity: 19952002. Pediatrics. 2005;116(3):580-6.

66. Orioli IM, Lima do Nascimento R, López-Camelo JS, Castilla EE. Effects of folic acid fortification on spina bifida prevalence in Brazil. Birth Defects Res A Clin Mol Teratol. 2011;91(9):831-5.

67. Bulbul A, Can E, Bulbul LG, Cömert S, Nuhoglu A. Clinical characteristics of neonatal meningomyelocele cases and effect of operation time on mortality and morbidity. Pediatr Neurosurg. 2010;46(3):199-204.

68. Tulipan N, Sutton LN, Bruner JP, Cohen BM, Johnson M, Adzick NS. The effect of intrauterine myelomeningocele repair on the incidence of shuntdependent hydrocephalus. Pediatr Neurosurg. 2003;38(1):27-33.

69. Fletcher JM, Copeland K, Frederick JA, Blaser SE, Kramer LA, Northrup H, Hannay HJ, Brandt ME, Francis DJ, Villarreal G, Drake JM, Laurent JP, Townsend I, Inwood S, Boudousquie A, Dennis M. Spinal lesion level in spina bifida: a source of neural and cognitive heterogeneity. $J$ Neurosurg. 2005;102(3 Suppl):268-79.

70. Charney EB, Melchionni JB, Antonucci DL. Ventriculitis in newborns with myelomeningocele. Am J Dis Child. 1991;145(3):287-90.

71. Demir N, Peker E, Gülşen I, Ağengin K, Tuncer O. Factors affecting infection development after meningomyelocele repair in newborns and the efficacy of antibiotic prophylaxis. Childs Nerv Syst. 2015;31(8):1355-9.

72. Holinger PC, Holinger LD, Reichert TJ, Holinger PH. Respiratory obstruction and apnea in infants with bilateral abductor vocal cord paralysis, meningomyelocele, hydrocephalus, and Arnold-Chiari malformation. $J$ Pediatr. 1978;92(3):368-73. 
73. da Silva SA, de Almeida MF, Moron AF, Cavalheiro S, Dastoli PA, Guinsburg R. Resuscitation at birth in neonates with meningomyelocele. $J$ Perinat Med. 2014;42(1):113-9.

74. Oi S, Di Rocco C. Proposal of "evolution theory in cerebrospinal fluid dynamics" and minor pathway hydrocephalus in developing immature brain. Childs Nerv Syst. 2006;22(7):662-9.

75. Babcook CJ, Goldstein RB, Barth RA, Damato NM, Callen PW, Filly RA. Prevalence of ventriculomegaly in association with myelomeningocele: correlation with gestational age and severity of posterior fossa deformity. Radiology. 1994;190(3):703-7.

76. Tamburrini G, Frassanito P, lakovaki K, Pignotti F, Rendeli C, Murolo D, Di Rocco C. Myelomeningocele: the management of the associated hydrocephalus. Childs Nerv Syst. 2013;29(9):1569-79.

77. Cuppen I, Eggink AJ, Lotgering FK, Rotteveel JJ, Mullaart RA, Roeleveld N. Influence of birth mode on early neurological outcome in infants with myelomeningocele. Eur J Obstet Gynecol Reprod Biol. 2011;156(1):18-22.

78. Wilson RD, Johnson MP, Bebbington M, Flake AW, Hedrick HL, Sutton LN, Adzick NS. Does a myelomeningocele sac compared to no sac result in decreased postnatal leg function following maternal fetal surgery for spina bifida aperta? Fetal Diagn Ther. 2007;22(5):348-51.

79. Oncel MY, Ozdemir R, Kahilogulları G, Yurttutan S, Erdeve O, Dilmen U. The effect of surgery time on prognosis in newborns with meningomyelocele. J Korean Neurosurg Soc. 2012;51(6):359-62. 